Bull. Soc. math. France

129 (2), 2001, p. 285-316

\title{
PROFILE DECOMPOSITION FOR SOLUTIONS OF THE NAVIER-STOKES EQUATIONS
}

\author{
By Isabelle Gallagher
}

\begin{abstract}
We consider sequences of solutions of the Navier-Stokes equations in $\mathbb{R}^{3}$, associated with sequences of initial data bounded in $\dot{H}^{1 / 2}$. We prove, in the spirit of the work of H. Bahouri and P. Gérard (in the case of the wave equation), that they can be decomposed into a sum of orthogonal profiles, bounded in $\dot{H}^{1 / 2}$, up to a remainder term small in $L^{3}$; the method is based on the proof of a similar result for the heat equation, followed by a perturbation-type argument. If $\mathcal{A}$ is an "admissible" space (in particular $L^{3}, \dot{B}_{p, \infty}^{-1+3 / p}$ for $p<+\infty$ or $\left.\nabla B M O\right)$, and if $\mathcal{B}_{N S}^{\mathcal{A}}$ is the largest ball in $\mathcal{A}$ centered at zero such that the elements of $\dot{H}^{1 / 2} \cap \mathcal{B}_{N S}^{\mathcal{A}}$ generate global solutions, then we obtain as a corollary an a priori estimate for those solutions. We also prove that the mapping from data in $\dot{H}^{1 / 2} \cap \mathcal{B}_{N S}^{\mathcal{A}}$ to the associate solution is Lipschitz.
\end{abstract}

Texte reçu le 6 juillet 2000, accepté le 13 octobre 2000

Isabelle Gallagher, Département de Mathématiques, UMR 8628, Université Paris-Sud, 91405 Orsay Cedex (France), Adresse actuelle : Centre de Mathématiques, École Polytechnique, 91128, Palaiseau CEDEx (France).

E-mail : Isabelle.Gallagher@math.polytechnique.fr

2000 Mathematics Subject Classification. - 35B45, 35Q30, 76D05.

Key words and phrases. - Navier-Stokes, explosion, profiles, a priori estimate, admissible space. 
RÉSUMÉ (Décomposition en profils pour les solutions des équations de Navier-Stokes)

On considère des suites de solutions des équations de Navier-Stokes dans $\mathbb{R}^{3}$, associées à des suites de données initiales bornées dans $\dot{H}^{1 / 2}$. On montre, dans l'esprit du travail de H. Bahouri et P. Gérard (dans le cas de l'équation des ondes), qu'elles peuvent être décomposées en une somme de profils orthogonaux, bornés dans $\dot{H}^{1 / 2}$, à un terme de reste près, petit dans $L^{3}$; la méthode s'appuie sur la démonstration d'un résultat analogue pour l'équation de la chaleur, suivi d'un argument de perturbation. Si $\mathcal{A}$ est un espace « admissible» (en particulier $L^{3}, \dot{B}_{p, \infty}^{-1+3 / p}$ pour $p<+\infty$ ou $\nabla B M O)$, et si $\mathcal{B}_{N S}^{\mathcal{A}}$ est la plus grande boule de de $\mathcal{A}$ centrée en zéro, telle que les éléments de $\dot{H}^{1 / 2} \cap \mathcal{B}_{N S}^{\mathcal{A}}$ génèrent des solutions globales, alors on obtient en corollaire une estimation a priori pour ces solutions. On montre aussi que l'application associant une donnée dans $\dot{H}^{1 / 2} \cap \mathcal{B}_{N S}^{\mathcal{A}}$ à sa solution est lipschitzienne.

\section{Introduction}

We are interested in the incompressible Navier-Stokes equations in three space dimensions

$$
\left\{\begin{array}{l}
\partial_{t} v+v \cdot \nabla v-\nu \Delta v=-\nabla p \quad \text { in } \mathbb{R}_{t}^{+} \times \mathbb{R}_{x}^{3} \\
\operatorname{div} v=0 \text { in } \mathbb{R}_{t}^{+} \times \mathbb{R}_{x}^{3} \\
v_{\mid t=0}=v_{0}
\end{array}\right.
$$

where $v_{0}$ is a divergence free vector field, $v(t, x)$ and $p(t, x)$ are respectively the velocity and the pressure fields of the fluid, and $\nu>0$ is the viscosity. The velocity is a three-component vector field, and the pressure is a scalar field. The divergence free condition on $v$ represents the incompressibility of the fluid. Here $t$ and $x$ are respectively the time and the space variables, with $t \in \mathbb{R}^{+}$and $x \in \mathbb{R}^{3}$. All the results stated here hold in the more general case of $x \in \mathbb{R}^{d}, d \geq 3$, with obvious adaptations, namely in the orders of the functional spaces considered.

In order to motivate our study, let us recall a few well-known facts concerning the system (NS). The most important results about the Cauchy problem were obtained by J. Leray in [21], who proved that for divergence free data $v_{0} \in$ $L^{2}\left(\mathbb{R}^{3}\right)$, there is a global, weak solution $v$ of $(\mathrm{NS})$ with

$$
v \in L^{\infty}\left(\mathbb{R}^{+}, L^{2}\left(\mathbb{R}^{3}\right)\right) \cap L^{2}\left(\mathbb{R}^{+}, \dot{H}^{1}\left(\mathbb{R}^{3}\right)\right),
$$

where $L^{p}\left(\mathbb{R}^{3}\right)$ denotes the usual Lebesgue space of order $p$, and where we have noted $\dot{H}^{s}\left(\mathbb{R}^{3}\right)$ the homogeneous Sobolev space of order $s$, defined by

$$
\forall s<\frac{3}{2}, \quad \dot{H}^{s}\left(\mathbb{R}^{3}\right) \stackrel{\text { def }}{=}\left\{u \in \mathcal{S}^{\prime}\left(\mathbb{R}^{3}\right) ;\|u\|_{\dot{H}^{s}\left(\mathbb{R}^{3}\right)}<+\infty\right\},
$$

where

$$
\|u\|_{\dot{H}^{s}\left(\mathbb{R}^{3}\right)} \stackrel{\text { def }}{=}\left(\int_{\mathbb{R}^{3}}|\xi|^{2 s}|\widehat{u}(\xi)|^{2} \mathrm{~d} \xi\right)^{1 / 2},
$$

TOME $129-2001-\mathrm{N}^{\mathrm{O}} 2$ 
and $\widehat{u}$ is the Fourier transform of $u$. We will note $(\cdot \mid \cdot)_{\dot{H}^{s}\left(\mathbb{R}^{3}\right)}$ the scalar product in $\dot{H}^{s}\left(\mathbb{R}^{3}\right)$. The restriction $s<\frac{3}{2}$ is for $\|u\|_{\dot{H}^{s}\left(\mathbb{R}^{3}\right)}$ to be a norm and not a semi-norm. Note that the inhomogeneous Sobolev space $H^{s}\left(\mathbb{R}^{3}\right)$ is of course defined in the same way, where $|\xi|^{2 s}$ is replaced by $\left(1+|\xi|^{2}\right)^{s}$. In the following, we will call $\dot{H}^{3 / 2}\left(\mathbb{R}^{3}\right)$ the space of vector fields whose components have first derivatives in $\dot{H}^{1 / 2}\left(\mathbb{R}^{3}\right)$.

The solutions constructed by J. Leray satisfy moreover the energy inequality

$$
\forall t \geq 0, \quad\|v(t)\|_{L^{2}\left(\mathbb{R}^{3}\right)}^{2}+2 \nu \int_{0}^{t}\|\nabla v(s)\|_{L^{2}\left(\mathbb{R}^{3}\right)}^{2} \mathrm{~d} s \leq\left\|v_{0}\right\|_{L^{2}\left(\mathbb{R}^{3}\right)}^{2} .
$$

Those solutions are not known to be unique (except in two space dimensions); many studies exist on that problem of uniqueness, and the starting point of our study will be the result of H. Fujita and T. Kato [8]. It can be stated in the following way (see [4] for instance): if $v_{0}$ is in $\dot{H}^{1 / 2}\left(\mathbb{R}^{3}\right)$, then there exists a unique maximal time $T_{*}>0$ and a unique solution $v$ associated with $v_{0}$ such that

$$
v \in C^{0}\left([0, T], \dot{H}^{1 / 2}\left(\mathbb{R}^{3}\right)\right) \cap L^{2}\left([0, T], \dot{H}^{3 / 2}\left(\mathbb{R}^{3}\right)\right) \quad \text { for all } T<T_{*} .
$$

Moreover, if $T_{*}<+\infty$, then we have

$$
\lim _{T \rightarrow T_{*}}\|v\|_{L^{2}\left([0, T], \dot{H}^{3 / 2}\left(\mathbb{R}^{3}\right)\right)}=+\infty .
$$

Furthermore, there exists a universal constant $c$ such that

$$
\left\|v_{0}\right\|_{\dot{H}^{1 / 2}\left(\mathbb{R}^{3}\right)} \leq c \nu \quad \Longrightarrow \quad T_{*}=+\infty
$$

and we have in that case, for any $t \geq 0$,

$$
\|v(t)\|_{\dot{H}^{1 / 2}\left(\mathbb{R}^{3}\right)}^{2}+\nu \int_{0}^{t}\|v(s)\|_{\dot{H}^{3 / 2}\left(\mathbb{R}^{3}\right)}^{2} \mathrm{~d} s \leq\left\|v_{0}\right\|_{\dot{H}^{1 / 2}\left(\mathbb{R}^{3}\right)}^{2} .
$$

Finally it is well known (see for instance [21] or [7], Remark 10.3 (a)) that we have the following weak-strong uniqueness result:

$$
\forall v_{0} \in L^{2} \cap \dot{H}^{1 / 2}\left(\mathbb{R}^{3}\right), \quad N S\left(v_{0}\right) \text { satisfies (1.1), }
$$

where, as in the whole of this text, we have noted $N S\left(v_{0}\right)$ the unique solution of (NS) associated with the initial data $v_{0} \in \dot{H}^{1 / 2}\left(\mathbb{R}^{3}\right)$.

One important aspect to keep in mind in the study of (NS) is the scaling of the equation. It is easy to check the following property: for any real number $\lambda$,

$$
v=N S\left(v_{0}\right) \quad \Longleftrightarrow \quad v_{\lambda}=N S\left(v_{0, \lambda}\right),
$$

with

$$
v_{\lambda}(t, x) \stackrel{\text { def }}{=} \lambda v\left(\lambda^{2} t, \lambda x\right) \quad \text { and } \quad v_{0, \lambda}(x) \stackrel{\text { def }}{=} \lambda v_{0}(\lambda x) .
$$

Note that the $\dot{H}^{1 / 2}\left(\mathbb{R}^{3}\right)$ norm is clearly conserved under the transformation $v_{0} \mapsto v_{0, \lambda}$. Many existence and uniqueness results have been obtained for data in such function spaces, invariant under that transformation; it is impossible to 
present here all the function spaces in which such results have been obtained, so let us simply recall the chain of spaces

$$
\dot{H}^{1 / 2}\left(\mathbb{R}^{3}\right) \subset L^{3}\left(\mathbb{R}^{3}\right) \subset \dot{B}_{p, \infty}^{-1+3 / p}\left(\mathbb{R}^{3}\right)_{\mid p<+\infty} \subset \nabla B M O\left(\mathbb{R}^{3}\right) \subset \dot{C}^{-1}\left(\mathbb{R}^{3}\right) .
$$

In that chain of spaces, $\dot{B}_{p, \infty}^{-1+3 / p}\left(\mathbb{R}^{3}\right)$ stands for a homogeneous Besov space. We shall not be using those spaces explicitly in this paper, so we will merely recall the following definition, using Littlewood Paley theory, and we refer for instance to [5] for a detailed presentation of the theory, and to [22] or [25] for the analysis of Besov spaces: elements of $\dot{B}_{p, \infty}^{s}\left(\mathbb{R}^{3}\right)$ satisfy

$$
\|u\|_{\dot{B}_{p, \infty}^{s}\left(\mathbb{R}^{3}\right)} \stackrel{\text { def }}{=} \sup _{j \in \mathbb{Z}} 2^{j s}\left\|\Delta_{j} u\right\|_{L^{p}\left(\mathbb{R}^{3}\right)}<+\infty,
$$

where $\Delta_{j}$ is a Littlewood-Paley operator, defined by

$$
\widehat{\Delta_{j} u}(\xi) \stackrel{\text { def }}{=} \varphi\left(2^{-j}|\xi|\right) \widehat{u}(\xi)
$$

and $\varphi \in \mathcal{C}_{c}^{\infty}\left(\left[\frac{1}{2}, 2\right]\right)$ satisfies $\sum_{j \in \mathbb{Z}} \varphi\left(2^{-j} t\right)=1$, for all $t>0$.

Furthermore, $\nabla B M O\left(\mathbb{R}^{3}\right)$ stands for the space of functions which are first derivatives of functions in $B M O\left(\mathbb{R}^{3}\right)$. We recall below the definition of the norm $\|u\|_{B M O\left(\mathbb{R}^{3}\right)}$, and refer to [24] for a detailed presentation of that space:

$$
\|u\|_{B M O\left(\mathbb{R}^{3}\right)} \stackrel{\text { def }}{=} \sup _{x_{0}, R} \frac{1}{\left|B\left(x_{0}, R\right)\right|} \int_{B\left(x_{0}, R\right)}\left|u-u_{B\left(x_{0}, R\right)}\right| \mathrm{d} x
$$

where

$$
u_{B\left(x_{0}, R\right)} \stackrel{\text { def }}{=} \frac{1}{\left|B\left(x_{0}, R\right)\right|} \int_{B\left(x_{0}, R\right)} u(x) \mathrm{d} x .
$$

In all those spaces except for the last, analogous existence and uniqueness theorems to the case $\dot{H}^{1 / 2}\left(\mathbb{R}^{3}\right)$ have been proved. We refer respectively to T. Kato [18] and G. Furioli, P.-G. Lemarié and E. Terraneo [10] for the proof of the $L^{3}\left(\mathbb{R}^{3}\right)$ case, to the book of M. Cannone [3] and the work of F. Planchon [23] for $\dot{B}_{p, \infty}^{-1+3 / p}\left(\mathbb{R}^{3}\right), p<+\infty$, and finally to H. Koch and D. Tataru [20] for the space $\nabla B M O$. In the space $\dot{C}^{-1}\left(\mathbb{R}^{3}\right) \stackrel{\text { def }}{=} \dot{B}_{\infty, \infty}^{-1}\left(\mathbb{R}^{3}\right)$, uniqueness was proved by J.-Y. Chemin in [6], supposing the data is also in the energy space $L^{2}\left(\mathbb{R}^{3}\right)$.

In relation with the result of $\mathrm{H}$. Fujita and T. Kato mentionned above, let us give the following definitions: we define the function spaces

$$
\left\{\begin{array}{l}
E_{T} \stackrel{\text { def }}{=} C^{0}\left([0, T], \dot{H}^{1 / 2}\left(\mathbb{R}^{3}\right)\right) \cap L^{2}\left([0, T], \dot{H}^{3 / 2}\left(\mathbb{R}^{3}\right)\right), \\
E_{\infty} \stackrel{\text { def }}{=} C_{b}^{0}\left(\mathbb{R}^{+}, \dot{H}^{1 / 2}\left(\mathbb{R}^{3}\right)\right) \cap L^{2}\left(\mathbb{R}^{+}, \dot{H}^{3 / 2}\left(\mathbb{R}^{3}\right)\right),
\end{array}\right.
$$

where $C_{b}^{0}$ denotes the set of bounded, continuous functions; we also define the sets of initial data yielding solutions of (NS) in $E_{T}$ and $E_{\infty}$ respectively,

$$
\begin{gathered}
\mathcal{D}_{T} \stackrel{\text { def }}{=}\left\{v_{0} \in \dot{H}^{1 / 2}\left(\mathbb{R}^{3}\right) \mid N S\left(v_{0}\right) \in E_{T}\right\}, \\
\mathcal{D}_{\infty} \stackrel{\text { def }}{=}\left\{v_{0} \in \dot{H}^{1 / 2}\left(\mathbb{R}^{3}\right) \mid N S\left(v_{0}\right) \in E_{\infty}\right\} .
\end{gathered}
$$

TOME $129-2001-\mathrm{N}^{\mathrm{O}} 2$ 
Finally we define, for any vector field $v$,

$$
\left\{\begin{array}{l}
\|v\|_{E_{T}^{\nu}} \stackrel{\text { def }}{=} \sup _{t \leq T}\left(\|v(t)\|_{\dot{H}^{1 / 2}\left(\mathbb{R}^{3}\right)}^{2}+2 \nu\|v\|_{L^{2}\left([0, t], \dot{H}^{3 / 2}\left(\mathbb{R}^{3}\right)\right)}^{2}\right)^{1 / 2}, \\
\|v\|_{E_{\infty}^{\nu}} \stackrel{\text { def }}{=}\left(\|v\|_{L^{\infty}\left(\mathbb{R}^{+}, \dot{H}^{1 / 2}\left(\mathbb{R}^{3}\right)\right)}^{2}+2 \nu\|v\|_{L^{2}\left(\mathbb{R}^{+}, \dot{H}^{3 / 2}\left(\mathbb{R}^{3}\right)\right)}^{2}\right)^{1 / 2} .
\end{array}\right.
$$

REMARK. - Note that nothing prevents a priori the life span $T_{*}$ associated with some data $v_{0}$ to satisfy $T_{*}=+\infty$ with $v_{0} \notin \mathcal{D}_{\infty}$ : in that case,

$$
\lim _{T \rightarrow+\infty}\left\|N S\left(v_{0}\right)\right\|_{L^{2}\left([0, T], \dot{H}^{3 / 2}\left(\mathbb{R}^{3}\right)\right)}=+\infty .
$$

Definition 1. - Let $\mathcal{A} \subset \mathcal{S}^{\prime}\left(\mathbb{R}^{3}\right)$ be a Banach space such that the embedding $\dot{H}^{1 / 2}\left(\mathbb{R}^{3}\right) \subset \mathcal{A}$ is continuous. Then $\mathcal{A}$ is admissible if and only if the following properties hold:

(i) The norm \|\|$_{\mathcal{A}}$ is invariant under the transformations

$$
\varphi \longmapsto \lambda \varphi(\lambda \cdot) \quad \forall \lambda \in \mathbb{R} \quad \text { and } \varphi \mapsto \varphi\left(\cdot-x_{0}\right) \quad \forall x_{0} \in \mathbb{R}^{3} .
$$

(ii) There exists a constant $c_{\nu}^{\mathcal{A}}$ depending only on $\nu$ and $\mathcal{A}$ such that if $\varphi$ is an element of $\dot{H}^{1 / 2}\left(\mathbb{R}^{3}\right)$ and $\|\varphi\|_{\mathcal{A}}$ is smaller than $c_{\nu}^{\mathcal{A}}$, then $\varphi$ is in $\mathcal{D}_{\infty}$.

ExAmple 1. - An obvious example is of course $\dot{H}^{1 / 2}\left(\mathbb{R}^{3}\right)$; point (i) is clear, and point (ii) is due to H. Fujita and T. Kato's theorem recalled above.

Example 2. - Similarly $L^{3}\left(\mathbb{R}^{3}\right)$ satisfies point (i), and a proof of (ii) can be found in Proposition A.1 in the Appendix.

ExAmple 3. - The Besov space $\dot{B}_{p, \infty}^{-1+3 / p}\left(\mathbb{R}^{3}\right)$ satisfies point (i), and point (ii) is proved for instance in Theorem 3.4.2 of [3] for $p<+\infty$.

ExAMPLE 4. - The space $\nabla B M O\left(\mathbb{R}^{3}\right)$ is a Banach space satisfying points (i) and (ii), as proved in [9].

In the following, for any admissible space $\mathcal{A}$ in the sense of Definition 1 , we shall define the constant $C_{N S}^{\mathcal{A}} \in \mathbb{R}^{+} \cup\{+\infty\}$ by

$$
C_{N S}^{\mathcal{A}} \stackrel{\text { def }}{=} \sup \left\{\rho>0 ; \overline{\mathcal{B}}_{\rho}^{\mathcal{A}} \cap \dot{H}^{1 / 2}\left(\mathbb{R}^{3}\right) \subset \mathcal{D}_{\infty}\right\},
$$

where

and we will note

$$
\mathcal{B}_{\rho}^{\mathcal{A}} \stackrel{\text { def }}{=}\left\{\varphi \in \mathcal{A} ;\|\varphi\|_{\mathcal{A}}<\rho\right\}
$$

$$
\mathcal{B}_{N S}^{\mathcal{A}} \stackrel{\text { def }}{=} \mathcal{B}_{C_{N S}}^{\mathcal{A}} \text {. }
$$

In other words, the set $\mathcal{B}_{N S}^{\mathcal{A}}$ is the largest ball in $\mathcal{A}$ whose intersection with $\dot{H}^{1 / 2}\left(\mathbb{R}^{3}\right)$ is a subset of $\mathcal{D}_{\infty}$. Note that we obviously have $C_{N S}^{\mathcal{A}} \geq c_{\nu}^{\mathcal{A}}$, where $c_{\nu}^{\mathcal{A}}$ was defined in Property (ii) of Definition 1. The following result will be proved in the Appendix. 
Proposition 1.1. - Let $v_{0} \in H^{1 / 2}\left(\mathbb{R}^{3}\right)$ be a divergence free vector field, and let $T_{*}$ be the life span of $N S\left(v_{0}\right)$. If $v_{0} \notin \mathcal{D}_{\infty}$, then

$$
T_{*} \leq \frac{1}{\nu\left(C_{N S}^{\dot{H}^{1 / 2}}\right)^{4}}\left\|v_{0}\right\|_{L^{2}\left(\mathbb{R}^{3}\right)}^{4} .
$$

Now let us come to the point of this study: it is well known that the embedding of $\dot{H}^{1 / 2}\left(\mathbb{R}^{3}\right)$ into $L^{3}\left(\mathbb{R}^{3}\right)$ is continuous, but not compact. Let us suppose for a moment that the embedding were in fact compact. If $\left(\varphi_{n}\right)$ is a bounded sequence of functions in $\dot{H}^{1 / 2}\left(\mathbb{R}^{3}\right)$, converging weakly to zero in $\dot{H}^{1 / 2}\left(\mathbb{R}^{3}\right)$, then it would converge strongly to zero in $L^{3}\left(\mathbb{R}^{3}\right)$; as a consequence, for $n$ large enough, the function $\varphi_{n}$ would be small enough for us to obtain a global, unique solution of (NS) in $E_{\infty}$ according to point (ii) of Definition 1 , since $L^{3}\left(\mathbb{R}^{3}\right)$ is an admissible space according to Example 2. In other words, if the embedding of $\dot{H}^{1 / 2}\left(\mathbb{R}^{3}\right)$ into $L^{3}\left(\mathbb{R}^{3}\right)$ was compact, then one could associate with any bounded sequence of divergence free vector fields in $\dot{H}^{1 / 2}\left(\mathbb{R}^{3}\right)$, converging weakly to zero in $\dot{H}^{1 / 2}\left(\mathbb{R}^{3}\right)$, a unique sequence of global solutions of (NS) in $E_{\infty}$. That remark leads us naturally to the problem of the defect of compactness of the embedding of $\dot{H}^{1 / 2}\left(\mathbb{R}^{3}\right)$ into $L^{3}\left(\mathbb{R}^{3}\right)$, which was very precisely studied by P. Gérard in [16]. Let us recall the result of [16]. The Theorem holds of course more generally for the embedding of $H^{s}\left(\mathbb{R}^{d}\right)$ into $L^{p}\left(\mathbb{R}^{d}\right)$ with $s=d\left(\frac{1}{2}-\frac{1}{p}\right)$.

TheOREM 1 (P. Gérard, [16]). — Let $\left(\varphi_{n}\right)$ be a bounded sequence of functions in $\dot{H}^{1 / 2}\left(\mathbb{R}^{3}\right)$. Then up to the extraction of a subsequence, it can be decomposed in the following way:

$$
\forall \ell \in \mathbb{N} \backslash\{0\}, \quad \varphi_{n}(x)=\varphi^{0}(x)+\sum_{j=1}^{\ell} \frac{1}{h_{n}^{j}} \varphi^{j}\left(\frac{x-x_{n}^{j}}{h_{n}^{j}}\right)+\psi_{n}^{\ell}(x),
$$

where the functions $\varphi^{j}$ are in $\dot{H}^{1 / 2}\left(\mathbb{R}^{3}\right)$ for all $j \in \mathbb{N}$, where $\left(\psi_{n}^{\ell}\right)$ is a bounded sequence in $\dot{H}^{1 / 2}\left(\mathbb{R}^{3}\right)$ uniformly in $\ell \in \mathbb{N} \backslash\{0\}$, and satisfies

$$
\lim _{\ell \rightarrow \infty}\left(\limsup _{n \rightarrow \infty}\left\|\psi_{n}^{\ell}\right\|_{L^{3}\left(\mathbb{R}^{3}\right)}\right)=0,
$$

and where for any $j \in \mathbb{N} \backslash\{0\},\left(h_{n}^{j}, x_{n}^{j}\right)$ is a sequence in $\left(\mathbb{R}^{+} \backslash\{0\} \times \mathbb{R}^{3}\right)^{\mathbb{N}}$ with the following orthogonality property: for every integers $(j, k)$ such that $j \neq k$, we have

$$
\begin{cases}\text { either } & \lim _{n \rightarrow \infty}\left(\frac{h_{n}^{j}}{h_{n}^{k}}+\frac{h_{n}^{k}}{h_{n}^{j}}\right)=+\infty \\ \text { or } & h_{n}^{j}=h_{n}^{k} \text { and } \lim _{n \rightarrow \infty} \frac{\left|x_{n}^{j}-x_{n}^{k}\right|}{h_{n}^{j}}=+\infty .\end{cases}
$$

TOME $129-2001-\mathrm{N}^{\mathrm{O}} 2$ 
Finally we have for every $\ell \in \mathbb{N} \backslash\{0\}$,

$$
\left\|\varphi_{n}\right\|_{\dot{H}^{1 / 2}\left(\mathbb{R}^{3}\right)}^{2}=\sum_{j=0}^{\ell}\left\|\varphi^{j}\right\|_{\dot{H}^{1 / 2}\left(\mathbb{R}^{3}\right)}^{2}+\left\|\psi_{n}^{\ell}\right\|_{\dot{H}^{1 / 2}\left(\mathbb{R}^{3}\right)}^{2}+o(1),
$$

as $n$ goes to infinity.

REMARK. - (a) As we shall see in Section 3, if $\varphi_{n}$ is divergence free for all integers $n$, then the same goes for $\varphi, \varphi^{j}$ and $\psi_{n}^{\ell}$, for all integers $j, \ell$ and $n$.

(b) The sequences $h_{n}^{j}$ are called the scales of $\varphi_{n}$, the points $x_{n}^{j}$ are the cores of concentration, and the functions

$$
\varphi_{n}^{j}(x) \stackrel{\text { def }}{=} \frac{1}{h_{n}^{j}} \varphi^{j}\left(\frac{x-x_{n}^{j}}{h_{n}^{j}}\right)
$$

are the associate profiles.

(c) Note that, up to rescaling the functions $\varphi^{j}$, one can suppose that for every $j \in \mathbb{N} \backslash\{0\}$, either $h_{n}^{j}=1$ and $\lim _{n \rightarrow \infty}\left|x_{n}^{j}\right|=+\infty$, or $\lim _{n \rightarrow \infty} h_{n}^{j}$ is in $\{0, \infty\}$.

(d) To simplify the notation, we shall note in the following

$$
h_{n}^{0} \stackrel{\text { def }}{=} 1, \quad x_{n}^{0} \stackrel{\text { def }}{=} 0, \quad \text { and } \quad \varphi_{n}^{0}(x) \stackrel{\text { def }}{=} \varphi^{0}(x) .
$$

(e) Finally we remark that the function $\varphi^{j}$, for every $j \in \mathbb{N}$, is a weak limit point of the sequence $h_{n}^{j} \varphi_{n}\left(x_{n}^{j}+h_{n}^{j}\right.$ ) ) (see for instance [16], formula (4.3)).

Our aim in this study is to see how decomposition (1.11) is propagated by the Navier-Stokes equation. By analogy with the work [1]-[2] on the critical semilinear wave equation, we shall also consider the linear equation associated with (NS), that is to say the heat equation

$$
\left\{\begin{array}{l}
\partial_{t} u-\nu \Delta u=0 \quad \text { in } \quad \mathbb{R}_{t}^{+} \times \mathbb{R}_{x}^{3} \\
u_{\mid t=0}=u_{0}
\end{array}\right.
$$

Notation. - In the following, we shall denote $H\left(u_{0}\right)$ the solution of the heat equation $(\mathrm{H})$ associated with the data $u_{0}$.

Note that if $u_{0} \in \dot{H}^{1 / 2}\left(\mathbb{R}^{3}\right)$, then $H\left(u_{0}\right) \in E_{\infty}$, and the norm $E_{\infty}^{\nu}$ is conserved by the application $H$. Note that $(H)$ has also the scale-invariance (1.6).

The following theorem gives a decomposition of the family of solutions of the system (NS) in the case of data bounded in $\dot{H}^{1 / 2}\left(\mathbb{R}^{3}\right)$. The last statement of that theorem (result (iii)) concerns the case of data bounded in $\mathcal{B}_{N S}^{\mathcal{A}}$, where $\mathcal{A}$ is any admissible space in the sense of Definition 1 , and we will start with that case in the proof of the result: as we shall see, that case enables one to avoid life span problems. The general case will then be treated using (iii). 
THEOREM 2. - Let $\left(\varphi_{n}\right)$ be a family of divergence free vector fields, bounded in $\dot{H}^{1 / 2}\left(\mathbb{R}^{3}\right)$, and let $\varphi^{0} \in \dot{H}^{1 / 2}\left(\mathbb{R}^{3}\right)$ be any weak limit point of $\left(\varphi_{n}\right)$. Then up to the extraction of a subsequence and with the notation of Theorem 1 and (1.16), the following results hold, where we define

$$
v_{n} \stackrel{\text { def }}{=} N S\left(\varphi_{n}\right) \text { and } V^{j} \stackrel{\text { def }}{=} N S\left(\varphi^{j}\right)
$$

for every integer $j \in \mathbb{N}$.

(i) There exists a family $\left(T^{j}\right)_{j \in \mathbb{N}}$ of elements of $\mathbb{R}^{+} \cup\{+\infty\}$ and a finite subset $J \subset \mathbb{N}$ such that

$$
\forall j \in \mathbb{N}, V^{j} \in E_{T^{j}} \quad \text { and } \quad \forall j \in \mathbb{N} \backslash J, T^{j}=+\infty .
$$

Moreover, if

$$
\tau_{n} \stackrel{\text { def }}{=} \min _{j \in J}\left(h_{n}^{j}\right)^{2} T^{j}
$$

then $\left\|v_{n}\right\|_{E_{\tau_{n}}^{\nu}}$ is bounded and we have for every integer $n \in \mathbb{N}$, for all times $t \leq \tau_{n}$, for every $\ell \in \mathbb{N}$ and every $x \in \mathbb{R}^{3}$,

$$
v_{n}(t, x)=\sum_{j=0}^{\ell} \frac{1}{h_{n}^{j}} V^{j}\left(\frac{t}{\left(h_{n}^{j}\right)^{2}}, \frac{x-x_{n}^{j}}{h_{n}^{j}}\right)+w_{n}^{\ell}(t, x)+r_{n}^{\ell}(t, x),
$$

where $w_{n}^{\ell} \stackrel{\text { def }}{=} H\left(\psi_{n}^{\ell}\right)$ and where

$$
\left\{\begin{array}{l}
\lim _{\ell \rightarrow \infty}\left(\limsup _{n \rightarrow \infty}\left\|w_{n}^{\ell}\right\|_{L^{\infty}\left(\mathbb{R}^{+}, L^{3}\left(\mathbb{R}^{3}\right)\right)}\right)=0 \\
\lim _{\ell \rightarrow \infty}\left(\limsup _{n \rightarrow \infty}\left\|r_{n}^{\ell}\right\|_{E_{\tau_{n}}^{\nu}}\right)=0 .
\end{array}\right.
$$

(ii) If there exists a time $T \in \mathbb{R}^{+} \cup\{+\infty\}$ such that $\left(v_{n}\right)$ is bounded in $L^{2}\left([0, T], \dot{H}^{3 / 2}\left(\mathbb{R}^{3}\right)\right)$, then we have

$$
\forall n \in \mathbb{N}, \quad T \leq \min _{j \in J}\left(h_{n}^{j}\right)^{2} T^{j} .
$$

In particular, the results above are valid with $\tau_{n}=T$ and the small scales of concentration generate global solutions of $(N S)$ : if $\lim _{n \rightarrow \infty} h_{n}^{j}=0$, then $T^{j}=+\infty$.

(iii) Let $\mathcal{A}$ be an admissible space in the sense of Definition 1 . Let $\rho$ be any real number in $] 0, C_{N S}^{\mathcal{A}}\left[\right.$. If $\left\|\varphi_{n}\right\|_{\mathcal{A}} \leq \rho$, then $T^{j}=+\infty$ for every $j \in \mathbb{N}$ and the results above hold with $\tau_{n}=+\infty$.

REMARK. - Case (i) shows that the life span of $v_{n}$ is bounded from below by the smallest of the life spans of each profile in the decomposition (1.18). In particular, there is no phenomenon such as two initial profiles $\varphi_{n}^{j}$ interacting and generating a solution for a smaller time than that generated by each profile separately. Case (ii) is a converse statement: if $v_{n}$ has a uniform life span, then each profile generates a solution at least on that life span. Finally, note that case (iii) is by no means a consequence of case (ii) because we do not suppose 
in case (iii) that the sequence of solutions $\left(N S\left(\varphi_{n}\right)\right)$ is bounded in $E_{\infty}$ : each term of the sequence is an element of $E_{\infty}$ since the initial data is bounded in $\mathcal{B}_{N S}^{\mathcal{A}}$, but no assumption is made on the boundedness of $\left(N S\left(\varphi_{n}\right)\right)$. Such a bound can in fact be deduced from Corollary 1 below.

Theorem 2 will enable us to infer the following corollaries. The method of proof of those results follows closely the arguments of [2] and [14], as we shall see in Section 4.

The following result shows that there is an a priori estimate for the $E_{\infty}^{\nu}$ norm of all solutions of (NS) associated with data in $\mathcal{B}_{N S}^{\mathcal{A}}$, with notation (1.9)(1.10); that result is obvious in the case of small initial data in $\dot{H}^{1 / 2}\left(\mathbb{R}^{3}\right)$, as can be seen in estimate (1.4). A similar result was proved in [2] in the case of the critical semilinear wave equation for Strichartz norms.

Corollary 1. - Let $\mathcal{A}$ be any admissible space, in the sense of Definition 1. Then there exists a nondecreasing function $A$ from $\mathbb{R}^{+} \times\left[0, \mathcal{C}_{N S}^{\mathcal{A}}\left[\right.\right.$ to $\mathbb{R}^{+}$such that for any divergence free vector field $\varphi$ in $\mathcal{B}_{N S}^{\mathcal{A}}$, we have

$$
\|N S(\varphi)\|_{E_{\infty}^{\nu}} \leq A\left(\|\varphi\|_{\dot{H}^{1 / 2}\left(\mathbb{R}^{3}\right)},\|\varphi\|_{\mathcal{A}}\right) .
$$

REMARK. - Global existence theorems are often proved by exhibiting some a priori estimate. Corollary 1 shows that whatever the method used to prove global existence in a ball $\mathcal{B}_{N S}^{\mathcal{A}}$, there is an a priori estimate for the solutions.

The next corollary is a consequence of Corollary 1 . Note that as above, that result is not difficult to prove in the case of small initial data in, say, $\dot{H}^{1 / 2}\left(\mathbb{R}^{3}\right)$; its interest is that it extends to any ball $\mathcal{B}_{N S}^{\mathcal{A}}$. It is proved using methods similar to $[14]$ in the case of the wave equation.

Corollary 2. - For any admissible space $\mathcal{A}$ in the sense of Definition 1 , the application NS mapping elements of $\mathcal{B}_{N S}^{\mathcal{A}} \cap \dot{H}^{1 / 2}\left(\mathbb{R}^{3}\right)$ to the associate solution of (NS) is Lipschitz on bounded subsets of the space $\mathcal{B}_{N S}^{\mathcal{A}} \cap \dot{H}^{1 / 2}\left(\mathbb{R}^{3}\right)$.

In the next theorem, we consider the case when the data is additionnally bounded in $L^{2}\left(\mathbb{R}^{3}\right)$ : according to $(1.1)$ and $(1.5)$, it is natural to consider data which is also bounded in energy.

TheOREM 3 (Bounded energy solutions). - Under the assumptions of Theorem 2 , if additionnally the sequence $\left(\varphi_{n}\right)$ is bounded in $L^{2}\left(\mathbb{R}^{3}\right)$, then the result of Theorem 2 (i) holds, where the large scales have disappeared: for every $j \in \mathbb{N} \backslash\{0\}$,

$$
\text { either } h_{n}^{j}=1 \text { and } \lim _{n \rightarrow \infty}\left|x_{n}^{j}\right|=+\infty, \quad \text { or } \lim _{n \rightarrow \infty} h_{n}^{j}=0 \text {. }
$$

Moreover, if the sequence $\left(v_{n}\right)$ is bounded in $L^{2}\left([0, T], \dot{H}^{3 / 2}\left(\mathbb{R}^{3}\right)\right)$ for some $T$ in $\mathbb{R}^{+} \cup\{+\infty\}$, then there exists a finite subset $J \subset \mathbb{N}$ satisfying (1.17), such 
that

$$
\left\{\begin{array}{l}
\forall j \in J, h_{n}^{j}=1 \text { and either } \liminf _{n \rightarrow \infty} T_{*}\left(v_{n}\right) \geq \min _{j \in J}\left(T_{*}^{j}\right) \\
\text { or } T^{j}=+\infty \forall j \in \mathbb{N} \text { and }\left(v_{n}\right) \text { is bounded in } E_{\infty},
\end{array}\right.
$$

where we have noted $T_{*}\left(v_{n}\right)$ and $T_{*}^{j}$ the life spans respectively of $v_{n}$ and $V^{j}$.

REMARK. - One can wonder what remains of those results when the setting is periodic instead of the whole space $\mathbb{R}^{3}$. Some remarks on that case are given at the end of Section 3.

FINAL REMARK. - The idea of decomposing solutions of non linear equations in such a way stems from the work of H. Bahouri and P. Gérard in [1], [2] concerning the critical semilinear wave equation in $\mathbb{R}^{3}$ (see [14] for the case of an exterior domain). There are two main differences between those studies and this one. First, the smoothing effect of the heat equation implies that for the NavierStokes equations (as well as for the heat equation, see Section 3.2), there are no concentrations at times other than $t=0$, contrary to hyperbolic equations like the wave equation; so the extraction of cores and times of concentration is much easier here (it comes directly from the decomposition of the data). Second, we do not have at our disposal a global solution associated with arbitrary data, so the control of life spans is a problem here; that is not the case for the wave equation. Note that a similar result to Theorem 2 (in the case (iii)) was proved by S. Keraani in [19] in the context of the Schrödinger equations.

The structure of the paper is as follows.

In Section 2, we prove a few orthogonality results concerning the NavierStokes equations with profiles as initial data, which will be used in the proof of Theorem 2.

Section 3 is devoted to the proofs of Theorems 2 and 3, whereas Corollaries 1 and 2 are proved in Section 4.

In the Appendix are proved a few classical results on the Navier-Stokes equations used in the course of the study. We also prove Proposition 1.1 stated above.

Some of the results proved in this paper were presented in [13].

Acknowledgments. - The author is grateful to P. Gérard for many helpful discussions.

\section{The Navier-Stokes equations with profiles as initial data}

In this section, we are going to prove some orthogonality results for solutions of (NS) with initial data of the form

$$
\varphi_{n} \stackrel{\text { def }}{=} \frac{1}{h_{n}} \varphi\left(\frac{x-x_{n}}{h_{n}}\right)
$$

TOME $129-2001-\mathrm{N}^{\mathrm{O}} 2$ 
with $\left(h_{n}, x_{n}\right) \in\left(\mathbb{R}^{+} \backslash\{0\} \times \mathbb{R}^{3}\right)^{\mathbb{N}}$. Those results will be used in the proof of Theorem 2.

Note that those orthogonality results are closely linked to the notion of $h_{n^{-}}$ oscillatory sequences (see [2], [15]-[17] for details, as well as Section 3.3); similar results could be proved in the more general setting of $h_{n}$-oscillation, but we shall not go into those considerations here.

Proposition 2.1. - Let $T \in \mathbb{R}^{+} \cup\{+\infty\}$ be given, and let $\varphi^{1}$ and $\varphi^{2}$ be two divergence free vector fields, elements of $\mathcal{D}_{T}$. Let us consider two orthogonal sequences of $\left(\mathbb{R}^{+} \backslash\{0\} \times \mathbb{R}^{3}\right)^{\mathbb{N}}$ in the sense of $(1.13)$, called $\left(h_{n}^{1}, x_{n}^{1}\right)$ and $\left(h_{n}^{2}, x_{n}^{2}\right)$. Suppose for instance that $h_{n}^{1} \leq h_{n}^{2}$. Then with notation (1.15), we have the following orthogonality results:

$$
\lim _{n \rightarrow \infty} \sup _{t \in\left[0,\left(h_{n}^{1}\right)^{2} T\right]}\left(N S\left(\varphi_{n}^{1}\right)(t, \cdot) \mid N S\left(\varphi_{n}^{2}\right)(t, \cdot)\right)_{\dot{H}^{1 / 2}\left(\mathbb{R}^{3}\right)}=0,
$$

as well as

$$
\lim _{n \rightarrow \infty}\left(N S\left(\varphi_{n}^{1}\right) \mid N S\left(\varphi_{n}^{2}\right)\right)_{L^{2}\left(\left[0,\left(h_{n}^{1}\right)^{2} T\right], \dot{H}^{3 / 2}\left(\mathbb{R}^{3}\right)\right)}=0
$$

and

$$
\lim _{n \rightarrow \infty}\left\|N S\left(\varphi_{n}^{1}\right) N S\left(\varphi_{n}^{2}\right)\right\|_{L^{4}\left(\left[0,\left(h_{n}^{1}\right)^{2} T\right], L^{2}\left(\mathbb{R}^{3}\right)\right)}=0 .
$$

Proof of Proposition 2.1. - We know, by the scale-invariance of (NS) recalled in (1.6), that the solution of (NS) associated with the data $\varphi_{n}^{j}$ is given by

$$
\forall j \in\{1,2\}, \quad v_{n}^{j}(t, x) \stackrel{\text { def }}{=} N S\left(\varphi_{n}^{j}\right)(t, x)=\frac{1}{h_{n}^{j}} V^{j}\left(\frac{t}{\left(h_{n}^{j}\right)^{2}}, \frac{x-x_{n}^{j}}{h_{n}^{j}}\right),
$$

where $V^{j} \stackrel{\text { def }}{=} N S\left(\varphi^{j}\right)$.

Note that $V^{j} \in E_{T}$, so $v_{n}^{j} \in E_{\left(h_{n}^{j}\right)^{2} T}$, with notation (1.7).

The results have nothing to do with the fact that $V^{j}$ are solutions of (NS), all we shall need is $V^{j} \in E_{T}$; so we can suppose that the functions $V^{j}$ are, say, smooth and compactly supported, and we have

$$
\begin{aligned}
&\left(N S\left(\varphi_{n}^{1}\right)(t, \cdot) \mid N S\left(\varphi_{n}^{2}\right)(t, \cdot)\right)_{\dot{H}^{1 / 2}\left(\mathbb{R}^{3}\right)} \\
&=\int_{\mathbb{R}^{3}}\left(h_{n}^{1}\right)^{-3 / 2}\left(h_{n}^{2}\right)^{-3 / 2}\left(\Lambda^{1 / 2} V^{1}\right)\left(\frac{t}{\left(h_{n}^{1}\right)^{2}}, \frac{x-x_{n}^{1}}{h_{n}^{1}}\right) \\
&\left(\Lambda^{1 / 2} V^{2}\right)\left(\frac{t}{\left(h_{n}^{2}\right)^{2}}, \frac{x-x_{n}^{2}}{h_{n}^{2}}\right) \mathrm{d} x,
\end{aligned}
$$

where $\Lambda \stackrel{\text { def }}{=} \sqrt{-\Delta}$. Let us start by supposing that $\lim _{n \rightarrow \infty} h_{n}^{1} / h_{n}^{2}=0$. Then the change of variables

$$
x=x_{n}^{1}+h_{n}^{1} y, \quad t=\left(h_{n}^{1}\right)^{2} s
$$

BULLETIN DE LA SOCIÉTÉ MATHÉMATIQUe DE FRANCE 
yields after an easy computation

$$
\forall t \in\left[0,\left(h_{n}^{1}\right)^{2} T\right], \quad\left|\left(N S\left(\varphi_{n}^{1}\right)(t, \cdot) \mid N S\left(\varphi_{n}^{2}\right)(t, \cdot)\right)_{\dot{H}^{1 / 2}\left(\mathbb{R}^{3}\right)}\right|=O\left(h_{n}^{1} / h_{n}^{2}\right)^{3 / 2},
$$

which gives the result. The sequences $\left(h_{n}^{1}, x_{n}^{1}\right)$ and $\left(h_{n}^{2}, x_{n}^{2}\right)$ are orthogonal, in the sense of (1.13), and we have supposed that $h_{n}^{1} \leq h_{n}^{2}$. So if $\lim _{n \rightarrow \infty} h_{n}^{1} / h_{n}^{2} \neq 0$, then $h_{n}^{1}=h_{n}^{2}$. In that case, the change of variables $(2.4)$ gives, for any $t \geq 0$, the following estimate:

$$
\begin{aligned}
& \left(N S\left(\varphi_{n}^{1}\right)(t, \cdot) \mid N S\left(\varphi_{n}^{2}\right)(t, \cdot)\right)_{\dot{H}^{1 / 2}\left(\mathbb{R}^{3}\right)} \\
& \quad=\int_{\mathbb{R}^{3}}\left(\Lambda^{1 / 2} V^{1}\right)(s, y)\left(\Lambda^{1 / 2} V^{2}\right)\left(s, y+\frac{x_{n}^{1}-x_{n}^{2}}{h_{n}^{1}}\right) \mathrm{d} x .
\end{aligned}
$$

The result (2.1) is then proved by the orthogonality property (1.13), since we have supposed that $V^{2}$ is compactly supported. The arguments are identical for (2.2) and left to the reader. Now let us prove (2.3). We start by supposing that $\lim _{n \rightarrow \infty} h_{n}^{1} / h_{n}^{2}=0$. Then for any $t \in\left[0,\left(h_{n}^{1}\right)^{2} T\right]$, we have

$$
\begin{aligned}
& \left\|N S\left(\varphi_{n}^{1}\right) N S\left(\varphi_{n}^{2}\right)\right\|_{L^{4}\left([0, t], L^{2}\left(\mathbb{R}^{3}\right)\right)}^{4}=\left(h_{n}^{1} h_{n}^{2}\right)^{-2} \\
& \quad \times \int_{0}^{t}\left\{\int_{\mathbb{R}^{3}}\left|V^{1}\left(\frac{t}{\left(h_{n}^{1}\right)^{2}}, \frac{x-x_{n}^{1}}{h_{n}^{1}}\right)\right|^{2} \times\left|V^{2}\left(\frac{t}{\left(h_{n}^{2}\right)^{2}}, \frac{x-x_{n}^{2}}{h_{n}^{2}}\right)\right|^{2} \mathrm{~d} x\right\}^{2} \mathrm{~d} t .
\end{aligned}
$$

Then the change of variables (2.4) yields

$$
\left\|v_{n}^{1} v_{n}^{2}\right\|_{L^{4}\left([0, t], L^{2}\left(\mathbb{R}^{3}\right)\right)}=O\left(h_{n}^{1} / h_{n}^{2}\right)
$$

which gives the result.

In the case when $h_{n}^{1}=h_{n}^{2}$, the change of variables (2.4) implies that

$$
\begin{aligned}
& \left\|v_{n}^{1} v_{n}^{2}\right\|_{L^{4}\left(\left[0,\left(h_{n}^{1}\right)^{2} T\right], L^{2}\left(\mathbb{R}^{3}\right)\right)}^{4} \\
& \quad=\int_{0}^{T}\left\{\int_{\mathbb{R}^{3}}\left|V^{1}(s, y)\right|^{2} \times\left|V^{2}\left(s, y+\frac{x_{n}^{1}-x_{n}^{2}}{h_{n}^{2}}\right)\right|^{2} \mathrm{~d} y\right\}^{2} \mathrm{~d} s,
\end{aligned}
$$

and as $V^{2}$ is compactly supported, the result follows. That proves Proposition 2.1.

\section{Profile decompositions: proof of Theorems 2 and 3}

This section is devoted to the proof of Theorems 2 and 3 . We start by writing, in Section 3.1, the decomposition of the family of initial data; the following section is devoted to the proof of Theorem 2. In the first paragraph of that section, we deal with the heat equation $(\mathrm{H})$, and prove a similar decomposition to Theorem 2 for that equation; the arguments are straighforward, due to the fact that the equation is linear and to the presence of the diffusion operator. Theorem 2 is proved in the following paragraphs. Finally Theorem 3 is proved in Section 3.3, and Section 3.4 consists in a remark on the periodic case.

TOME $129-2001-\mathrm{N}^{\mathrm{O}} 2$ 
In order to make the discussion simpler, we shall neglect in the following to extract subsequences; all the arguments below are valid up to the extraction of a subsequence.

3.1. Introduction: decomposition of the data. - Let us consider a bounded family of divergence free vector fields $\left(\varphi_{n}\right)$ in $\dot{H}^{1 / 2}\left(\mathbb{R}^{3}\right)$, and let $\varphi^{0}$ be any weak limit point of $\left(\varphi_{n}\right)$ in $\dot{H}^{1 / 2}\left(\mathbb{R}^{3}\right)$. Then we can apply Theorem 1 to $\left(\varphi_{n}-\varphi^{0}\right)$, and we have, with notation $(1.16)$,

$$
\forall \ell \in \mathbb{N}, \quad \varphi_{n}(x)=\sum_{j=0}^{\ell} \frac{1}{h_{n}^{j}} \varphi^{j}\left(\frac{x-x_{n}^{j}}{h_{n}^{j}}\right)+\psi_{n}^{\ell}(x),
$$

where $\varphi^{j}$ is in $\dot{H}^{1 / 2}\left(\mathbb{R}^{3}\right)$ for $j \in \mathbb{N}$, where $\left(\psi_{n}^{\ell}\right)$ is a bounded sequence in $\dot{H}^{1 / 2}\left(\mathbb{R}^{3}\right)$ uniformly in $\ell \in \mathbb{N}$, and satisfies the limit (1.12). The sequences $\left(h_{n}^{j}, x_{n}^{j}\right)$ are orthogonal in the sense of (1.13), and finally for every $\ell \in \mathbb{N}$, we have orthogonality of the $\dot{H}^{1 / 2}\left(\mathbb{R}^{3}\right)$ norms written in (1.14).

Note that the remark (e) after the statement of Theorem 1, in the introduction, implies in particular that the functions $\varphi_{n}^{j}$ defined in (1.15) are divergence free.

Lemma 3.1. - The functions $\varphi^{j}$ of decomposition (3.1) satisfy

$$
\lim _{j \rightarrow \infty}\left\|\varphi^{j}\right\|_{\dot{H}^{1 / 2}\left(\mathbb{R}^{3}\right)}=0 \text {. }
$$

Proof of Lemma 3.1. - Equation (1.14) implies that the series of general term $\left\|\varphi^{j}\right\|_{\dot{H}^{1 / 2}\left(\mathbb{R}^{3}\right)}^{2}$ is convergent, which proves the result.

3.2. Proof of Theorem 2. - The goal of this section is to prove Theorem 2: we consider a sequence $\left(\varphi_{n}\right)$, bounded in $\dot{H}^{1 / 2}\left(\mathbb{R}^{3}\right)$, converging weakly towards $\varphi^{0} \in \dot{H}^{1 / 2}\left(\mathbb{R}^{3}\right)$. We start by proving a similar result to Theorem 2 in the case of the heat equation: the proof of that result is quite straightforward. Then, in the next paragraph, we deal with the Navier-Stokes equations. We first consider the case when the norm of $\varphi_{n}$ in some admissible space $\mathcal{A}$, in the sense of Definition 1, is smaller than the constant $C_{N S}^{\mathcal{A}}$, with notation (1.9); that enables us to avoid life span problems, and to prove Theorem 2 (iii). The general $\dot{H}^{1 / 2}\left(\mathbb{R}^{3}\right)$ case is treated in the following paragraph, using (iii).

3.2.1. A profile decomposition for the heat equation. - Let us prove the following result.

Proposition 3.2. - Let $\left(\varphi_{n}\right)$ be a family of divergence free vector fields, bounded in $\dot{H}^{1 / 2}\left(\mathbb{R}^{3}\right)$, and let $\varphi^{0} \in \dot{H}^{1 / 2}\left(\mathbb{R}^{3}\right)$ be any weak limit point of $\left(\varphi_{n}\right)$. Then up to the extraction of a subsequence, the following result holds, where with the notation of Theorem 1 and (1.16) we define

$$
u_{n} \stackrel{\text { def }}{=} H\left(\varphi_{n}\right) \in E_{\infty} \quad \text { and } \quad U^{j} \stackrel{\text { def }}{=} H\left(\varphi^{j}\right) \in E_{\infty}
$$

BULletin DE LA SOCIÉtÉ MATHÉMATIQUE DE FRANCE 
for every integer $j \in \mathbb{N}$.

For every $\ell \in \mathbb{N}$, for every $t \geq 0$ and every $x \in \mathbb{R}^{3}$, we can write

$$
u_{n}(t, x)=\sum_{j=0}^{\ell} \frac{1}{h_{n}^{j}} U^{j}\left(\frac{t}{\left(h_{n}^{j}\right)^{2}}, \frac{x-x_{n}^{j}}{h_{n}^{j}}\right)+w_{n}^{\ell}(t, x),
$$

where $w_{n}^{\ell} \stackrel{\text { def }}{=} H\left(\psi_{n}^{\ell}\right)$ is bounded in $E_{\infty}$ uniformly in $\ell \in \mathbb{N}$, with

$$
\lim _{\ell \rightarrow \infty}\left(\limsup _{n \rightarrow \infty}\left\|w_{n}^{\ell}\right\|_{L^{\infty}\left(\mathbb{R}^{+}, L^{3}\left(\mathbb{R}^{3}\right)\right)}\right)=0 .
$$

Moreover, the sequences $\left(h_{n}^{j}, x_{n}^{j}\right)$ are orthogonal in the sense of (1.13) and finally we have for every $\ell \in \mathbb{N}$,

$$
\left\|u_{n}\right\|_{E_{\infty}^{\nu}}^{2}=\sum_{j=0}^{\ell}\left\|U^{j}\right\|_{E_{\infty}^{\nu}}^{2}+\left\|w_{n}^{\ell}\right\|_{E_{\infty}^{\nu}}^{2}+o(1), \quad \text { when } n \rightarrow \infty .
$$

Proof of Proposition 3.2. - We consider decomposition (3.1), and we define

$$
\forall j \in \mathbb{N}, U^{j} \stackrel{\text { def }}{=} H\left(\varphi^{j}\right) \quad \text { and } \quad \forall(\ell, n) \in \mathbb{N}^{2}, w_{n}^{\ell} \stackrel{\text { def }}{=} H\left(\psi_{n}^{\ell}\right) .
$$

By the scale-invariance (1.6) of $(H)$, we have

$$
u_{n}^{j}(t, x) \stackrel{\text { def }}{=} \frac{1}{h_{n}^{j}} U^{j}\left(\frac{t}{\left(h_{n}^{j}\right)^{2}}, \frac{x-x_{n}^{j}}{h_{n}^{j}}\right)=H\left(\varphi_{n}^{j}\right),
$$

so we can write for every $(\ell, n) \in \mathbb{N}^{2}$,

$$
u_{n}=\sum_{j=0}^{\ell} u_{n}^{j}+w_{n}^{\ell}, \quad \text { where } \quad u_{n} \stackrel{\text { def }}{=} H\left(\varphi_{n}\right) .
$$

So all we need to check are properties (3.2) and (3.3).

The limit (3.2) is an immediate consequence of (1.12), since it is a wellknown fact (see for instance [3], Lemma 3.2.2 and formula (3.15)) that for any $u_{0} \in L^{3}\left(\mathbb{R}^{3}\right)$,

$$
\left\|H\left(u_{0}\right)\right\|_{L^{\infty}\left(\mathbb{R}^{+}, L^{3}\left(\mathbb{R}^{3}\right)\right)} \leq\left\|u_{0}\right\|_{L^{3}\left(\mathbb{R}^{3}\right)} .
$$

Finally the orthogonality result (3.3) is simply due to the fact that for any vector field $u_{0}$ in $\dot{H}^{1 / 2}\left(\mathbb{R}^{3}\right)$, we have

$$
\left\|H\left(u_{0}\right)\right\|_{E_{\infty}^{\nu}}=\left\|u_{0}\right\|_{\dot{H}^{1 / 2}\left(\mathbb{R}^{3}\right)} .
$$

Then (1.14), associated with the scale-invariance

$$
\forall(j, n) \in \mathbb{N}^{2}, \quad\left\|u_{n}^{j}\right\|_{E_{\infty}^{\nu}}=\left\|U^{j}\right\|_{E_{\infty}^{\nu}},
$$

yields (3.3). That proves Proposition 3.2.

TOME $129-2001-\mathrm{N}^{\mathrm{O}} 2$ 
REMARK. - As noted in the introduction, the presence of the diffusion operator $-\Delta$ prevents concentrations from occuring at another time than $t=0$; that can be seen in estimate (3.5). In the case of the wave equation [1]-[2], concentrations can occur at any time.

Now let us deal with the Navier-Stokes equations. Note that contrary to the case of the critical semilinear wave equation [1], [2] where the solutions are global in time, we are going to have to pay a careful attention to life spans. In the next paragraph, result (iii) of Theorem 2 is proved, in which there are no life span problems. That result will then help us prove the theorem.

3.2.2. Proof of Theorem $2($ iii $)$. — Let $\mathcal{A}$ be an admissible space, in the sense of Definition 1 , and let $\rho \in] 0, C_{N S}^{\mathcal{A}}$ [ be given, with notation (1.9), such that

$$
\forall n \in \mathbb{N}, \quad\left\|\varphi_{n}\right\|_{\mathcal{A}} \leq \rho
$$

Recall that for every $j \in \mathbb{N}$, with the notation of Theorem 1 , the function $\varphi^{j}$ is a weak limit point in $\dot{H}^{1 / 2}\left(\mathbb{R}^{3}\right)$ of the sequence $\Phi_{n}^{j} \stackrel{\text { def }}{=} h_{n}^{j} \varphi_{n}\left(x_{n}^{j}+h_{n}^{j} \cdot\right)$. By property (i) of Definition 1 , we have

$$
\forall(j, n) \in \mathbb{N}^{2}, \quad\left\|\varphi_{n}\right\|_{\mathcal{A}}=\left\|\Phi_{n}^{j}\right\|_{\mathcal{A}} .
$$

Moreover, since $\dot{H}^{1 / 2}\left(\mathbb{R}^{3}\right)$ is embedded in $\mathcal{A}$, we have $\varphi^{j} \in \mathcal{A}$, and since $\mathcal{A}$ is a Banach space, the results (3.6) and (3.7) imply that

$$
\forall j \in \mathbb{N}, \quad\left\|\varphi^{j}\right\|_{\mathcal{A}} \leq \rho .
$$

So in particular, by definition of $\rho$, we have $\varphi^{j} \in \mathcal{D}_{\infty}$ for all integers $j$, and as a consequence one can associate with $\varphi^{j}$ a global solution of the Navier-Stokes equations, namely

$$
\forall j \in \mathbb{N}, \quad V^{j} \stackrel{\text { def }}{=} N S\left(\varphi^{j}\right) \in E_{\infty},
$$

where recall that $E_{\infty}=C_{b}^{0}\left(\mathbb{R}^{+}, \dot{H}^{1 / 2}\left(\mathbb{R}^{3}\right)\right) \cap L^{2}\left(\mathbb{R}^{+}, \dot{H}^{3 / 2}\left(\mathbb{R}^{3}\right)\right)$. By the scale invariance of the Navier-Stokes equations explained in the introduction (see (1.6)), we know that

$$
v_{n}^{j}(t, x) \stackrel{\text { def }}{=} \frac{1}{h_{n}^{j}} V^{j}\left(\frac{t}{\left(h_{n}^{j}\right)^{2}}, \frac{x-x_{n}^{j}}{h_{n}^{j}}\right)
$$

is the unique, global solution of (NS) associated with the data $\varphi_{n}^{j}$. Now for every integer $\ell \in \mathbb{N}$, let us define

$$
r_{n}^{\ell} \stackrel{\text { def }}{=} v_{n}-\sum_{j \leq \ell} v_{n}^{j}-w_{n}^{\ell},
$$

where $w_{n}^{\ell}=H\left(\psi_{n}^{\ell}\right)$ as in Proposition 3.2, and $v_{n} \stackrel{\text { def }}{=} N S\left(\varphi_{n}\right)$. To prove the result, it is enough to prove that

$$
\lim _{\ell \rightarrow \infty}\left(\limsup _{n \rightarrow \infty} r_{n}^{\ell}\right)=0 \quad \text { in } \quad E_{\infty}
$$

BULletin DE LA SOCiÉtÉ MATHÉmATiQUe DE FRANCE 
The function $r_{n}^{\ell}$ satisfies the following system:

$$
\left\{\begin{array}{l}
\partial_{t} r_{n}^{\ell}+P\left(r_{n}^{\ell} \cdot \nabla r_{n}^{\ell}\right)-\nu \Delta r_{n}^{\ell}+Q\left(r_{n}^{\ell}, f_{n}^{\ell}\right)=g_{n}^{\ell} \quad \text { in } \mathbb{R}^{+} \times \mathbb{R}^{3}, \\
r_{n \mid t=0}^{\ell}=0,
\end{array}\right.
$$

where

$$
f_{n}^{\ell} \stackrel{\text { def }}{=} \sum_{j \leq \ell} v_{n}^{j}+w_{n}^{\ell}
$$

and

$$
g_{n}^{\ell} \stackrel{\text { def }}{=}-\frac{1}{2} \sum_{\substack{j \neq k \\(j, k) \in\{0, \ldots, \ell\}^{2}}} Q\left(v_{n}^{j}, v_{n}^{k}\right)-\sum_{j \leq \ell} Q\left(v_{n}^{j}, w_{n}^{\ell}\right)-P\left(w_{n}^{\ell} \cdot \nabla w_{n}^{\ell}\right),
$$

where

$$
Q(a, b) \stackrel{\text { def }}{=} P(a \cdot \nabla b+b \cdot \nabla a),
$$

and finally where $P$ is the orthogonal projector onto divergence-free vector fields.

Proposition 3.3. - With notation (3.12) and (3.13), we have the following results: the sequence $\left(f_{n}^{\ell}\right)$ is bounded in the space $E_{\infty}$, uniformly in $\ell$, and

$$
\lim _{\ell \rightarrow \infty} \limsup _{n \rightarrow \infty}\left\|g_{n}^{\ell}\right\|_{L^{2}\left(\mathbb{R}^{+}, \dot{H}^{-1 / 2}\left(\mathbb{R}^{3}\right)\right)}=0 .
$$

Let us postpone the proof of Proposition 3.3 and finish the proof of Theorem 2 (iii). By interpolation between the spaces $L^{\infty}\left(\mathbb{R}^{+}, \dot{H}^{1 / 2}\left(\mathbb{R}^{3}\right)\right)$ and $L^{2}\left(\mathbb{R}^{+}, \dot{H}^{3 / 2}\left(\mathbb{R}^{3}\right)\right)$, we know from Proposition 3.3 that $\left(f_{n}^{\ell}\right)$ is bounded in the space $L^{4}\left(\mathbb{R}^{+}, \dot{H}^{1}\left(\mathbb{R}^{3}\right)\right)$, uniformly in $\ell$. Proposition A.2 proved in the Appendix, applied to the sequence $r_{n}^{\ell}$, implies that for $\ell$ large enough, uniformly in $n$, we have according to Proposition 3.3

$$
\sup _{n \in \mathbb{N}}\left\|g_{n}^{\ell}\right\|_{L^{2}\left(\mathbb{R}^{+}, \dot{H}^{-1 / 2}\left(\mathbb{R}^{3}\right)\right)} \leq C \exp \left(-2 C \sup _{(\ell, n) \in \mathbb{N}^{2}}\left\|f_{n}^{\ell}\right\|_{L^{4}\left(\mathbb{R}^{+}, \dot{H}^{1}\left(\mathbb{R}^{3}\right)\right)}^{4}\right),
$$

hence we get

$$
\left\|r_{n}^{\ell}\right\|_{E_{\infty}^{\nu}} \leq C\left\|g_{n}^{\ell}\right\|_{L^{2}\left(\mathbb{R}^{+}, \dot{H}^{-1 / 2}\left(\mathbb{R}^{3}\right)\right)}\left(1+\exp \left(C\left\|f_{n}^{\ell}\right\|_{L^{4}\left(\mathbb{R}^{+}, \dot{H}^{1}\left(\mathbb{R}^{3}\right)\right.}^{4}\right)\right) .
$$

So (3.10) is proved, and with it, Theorem 2 (iii).

Proof of Proposition 3.3. - Let us start by noticing that the sequence $\left(v_{n}^{j}\right)$ is bounded in $E_{\infty}$ for every $j \in \mathbb{N}$ since

$$
\left\|v_{n}^{j}\right\|_{E_{\infty}^{\nu}}=\left\|V^{j}\right\|_{E_{\infty}^{\nu}} .
$$

Note however that we do not have at our disposal an a priori estimate for $\left\|v_{n}^{j}\right\|_{E_{\infty}^{\nu}}$ (such an a priori estimate is available for small initial data in $\dot{H}^{1 / 2}\left(\mathbb{R}^{3}\right.$ ) for instance (see (1.4)), but in the more general $\mathcal{B}_{N S}^{\mathcal{A}}$ case, it is a consequence of Corollary 1 proved later in this paper).

TOME $129-2001-\mathrm{N}^{\mathrm{O}} 2$ 
The following proposition will be proved later. Note that it is written in a general setting, and not only for sequences $\left(\varphi_{n}\right)$ in $\mathcal{B}_{N S}^{\mathcal{A}}$, because it will also be used in the proof of the general case of Theorem 2 , in the next section.

Proposition 3.4. - Let $\left(\varphi_{n}\right)$ be a bounded sequence of divergence free vector fields in $\dot{H}^{1 / 2}\left(\mathbb{R}^{3}\right)$, and let $\varphi^{0}$ be a weak limit point of $\left(\varphi_{n}\right)$. There exists an integer $j_{0}$ such that the following holds. Let $\left(\varphi^{j}\right)_{j \in \mathbb{N}}$ be the profiles of the decomposition of $\varphi_{n}$, with the notation of Theorem 1 , and let $V^{j} \stackrel{\text { def }}{=} N S\left(\varphi^{j}\right)$. Then we have

$$
\forall j \geq j_{0}, \quad V^{j} \in E_{\infty} \text { and } \sum_{j \geq j_{0}}\left\|V^{j}\right\|_{E_{\infty}^{\nu}}^{2}<+\infty
$$

From that result we can deduce the bound on $f_{n}^{\ell}$. We have indeed, from $(2.1)$ and (2.2) proved in Proposition 2.1,

$$
\forall \ell \in \mathbb{N}, \quad\left\|\sum_{j \leq \ell} v_{n}^{j}\right\|_{E_{\infty}^{\nu}}^{2}=\sum_{j \leq \ell}\left\|v_{n}^{j}\right\|_{E_{\infty}^{\nu}}^{2}+o(1), \quad \text { when } n \rightarrow \infty .
$$

By a change of scale, we get

$$
\left\|\sum_{j \leq \ell} v_{n}^{j}\right\|_{E_{\infty}^{\nu}}^{2}=\sum_{j \leq \ell}\left\|V^{j}\right\|_{E_{\infty}^{\nu}}^{2}+o(1), \quad \text { when } n \rightarrow \infty,
$$

hence Proposition 3.4, associated with the fact that $V^{j} \in E_{\infty}$ for every integer $j$ according to (3.8), implies that

$$
\sum_{j \leq \ell} v_{n}^{j} \text { is bounded in } E_{\infty}, \text { uniformly in } \ell .
$$

So the result on $f_{n}^{\ell}$ is proved.

Now let us prove the limit on $g_{n}^{\ell}$ : it is enough to prove the three following results:

$$
\begin{aligned}
& \forall j \neq k, \quad \lim _{n \rightarrow \infty} Q\left(v_{n}^{j}, v_{n}^{k}\right)=0 \quad \text { in } L^{4}\left(\mathbb{R}^{+}, \dot{H}^{-1}\left(\mathbb{R}^{3}\right)\right), \\
& \lim _{\ell \rightarrow \infty} \limsup _{n \rightarrow \infty} Q\left(\sum_{j \leq \ell} v_{n}^{j}, w_{n}^{\ell}\right)=0 \quad \text { in } L^{4}\left(\mathbb{R}^{+}, \dot{H}^{-1}\left(\mathbb{R}^{3}\right)\right), \\
& \lim _{\ell \rightarrow \infty}\left(\limsup _{n \rightarrow \infty} Q\left(w_{n}^{\ell}, w_{n}^{\ell}\right)\right)=0 \quad \text { in } L^{4}\left(\mathbb{R}^{+}, \dot{H}^{-1}\left(\mathbb{R}^{3}\right)\right) .
\end{aligned}
$$

Indeed, one can notice that the sequences $\left(v_{n}^{j}\right)$ and $\left(w_{n}^{\ell}\right)$ are bounded in $L^{8 / 3}\left(\mathbb{R}^{+}, \dot{H}^{5 / 4}\left(\mathbb{R}^{3}\right)\right)$ uniformly in $j$ and $\ell$, by interpolation between the space $L^{\infty}\left(\mathbb{R}^{+}, \dot{H}^{1 / 2}\left(\mathbb{R}^{3}\right)\right)$ and $L^{2}\left(\mathbb{R}^{+}, \dot{H}^{3 / 2}\left(\mathbb{R}^{3}\right)\right)$. Now let us recall the product rules in Sobolev spaces: if $a$ and $b$ are two tempered distributions, then

$$
\forall s, t<\frac{3}{2}, \quad s+t>0, \quad\|a b\|_{\dot{H}^{s+t-\frac{3}{2}\left(\mathbb{R}^{3}\right)}} \leq C_{s, t}\|a\|_{\dot{H}^{s}\left(\mathbb{R}^{3}\right)}\|b\|_{\dot{H}^{t}\left(\mathbb{R}^{3}\right)} .
$$

BULLETIN DE LA SOCiÉtÉ MATHÉMATIQUE DE FRANCE 
Those rules enable us to infer that $g_{n}^{\ell}$ is bounded in $L^{4 / 3}\left(\mathbb{R}^{+}, L^{2}\left(\mathbb{R}^{3}\right)\right)$. Then by interpolation with the results (3.16) to (3.18), the proposition is proved.

So let us prove those three limits: the product rules imply that the sequences $Q\left(v_{n}^{j}, v_{n}^{k}\right), Q\left(v_{n}^{j}, w_{n}^{\ell}\right)$ and $Q\left(w_{n}^{\ell}, w_{n}^{\ell}\right)$ are bounded in $L^{4}\left(\mathbb{R}^{+}, \dot{H}^{-1}\left(\mathbb{R}^{3}\right)\right)$ for every $(j, k, \ell) \in \mathbb{N}^{3}$, since by interpolation between $L^{\infty}\left(\mathbb{R}^{+}, \dot{H}^{1 / 2}\left(\mathbb{R}^{3}\right)\right)$ and $L^{2}\left(\mathbb{R}^{+}, \dot{H}^{3 / 2}\left(\mathbb{R}^{3}\right)\right)$, the functions $v_{n}^{j}$ and $w_{n}^{\ell}$ are also bounded in the space $L^{4}\left(\mathbb{R}^{+}, \dot{H}^{1}\left(\mathbb{R}^{3}\right)\right)$.

Now let us prove that for $j \neq k$, the function $Q\left(v_{n}^{j}, v_{n}^{k}\right)$ goes to zero in $L^{4}\left(\mathbb{R}^{+}, \dot{H}^{-1}\left(\mathbb{R}^{3}\right)\right)$ as $n$ goes to infinity. The divergence free condition on $v_{n}^{j}$ implies that

$$
P\left(v_{n}^{j} \cdot \nabla v_{n}^{k}\right)=P \operatorname{div}\left(v_{n}^{j} \otimes v_{n}^{k}\right),
$$

so since $P$ is a Fourier multiplier of order 0 , it is enough to prove that

$$
\forall j \neq k, \quad \lim _{n \rightarrow \infty}\left\|v_{n}^{j} v_{n}^{k}\right\|_{L^{4}\left(\mathbb{R}^{+}, L^{2}\left(\mathbb{R}^{3}\right)\right)}=0 .
$$

That result is simply due to (2.3) proved in Proposition 2.1 of the previous section, so we have obtained (3.16).

To obtain (3.17) and (3.18), the method is different since $w_{n}^{\ell}$ is not oscillatory, so we cannot use Proposition 2.1; that is compensated by the fact that, according to Proposition 3.2,

$$
\lim _{\ell \rightarrow \infty}\left(\limsup _{n \rightarrow \infty}\left\|w_{n}^{\ell}\right\|_{L^{\infty}\left(\mathbb{R}^{+}, L^{3}\left(\mathbb{R}^{3}\right)\right)}\right)=0 .
$$

As in (3.20), it is enough to prove that

$$
\lim _{\ell \rightarrow \infty} \limsup _{n \rightarrow \infty}\left\|\left(\sum_{j \leq \ell} v_{n}^{j}+w_{n}^{\ell}\right) w_{n}^{\ell}\right\|_{L^{4}\left(\mathbb{R}^{+}, L^{2}\left(\mathbb{R}^{3}\right)\right)}=0 .
$$

But Hölder's inequality yields

$$
\begin{aligned}
\|\left(\sum_{j \leq \ell} v_{n}^{j}\right. & \left.+w_{n}^{\ell}\right) w_{n}^{\ell} \|_{L^{4}\left(\mathbb{R}^{+}, L^{2}\left(\mathbb{R}^{3}\right)\right)} \\
& \leq\left\|\sum_{j \leq \ell} v_{n}^{j}+w_{n}^{\ell}\right\|_{L^{4}\left(\mathbb{R}^{+}, L^{6}\left(\mathbb{R}^{3}\right)\right)}\left\|w_{n}^{\ell}\right\|_{L^{\infty}\left(\mathbb{R}^{+}, L^{3}\left(\mathbb{R}^{3}\right)\right)} .
\end{aligned}
$$

Since $\dot{H}^{1}\left(\mathbb{R}^{3}\right)$ is embedded into $L^{6}\left(\mathbb{R}^{3}\right)$, we have for all $n \in \mathbb{N}$,

$$
\begin{aligned}
\left\|\sum_{j \leq \ell} v_{n}^{j}+w_{n}^{\ell}\right\|_{L^{4}\left(\mathbb{R}^{+}, L^{6}\left(\mathbb{R}^{3}\right)\right)} & \leq C\left\|\sum_{j \leq \ell} v_{n}^{j}+w_{n}^{\ell}\right\|_{L^{4}\left(\mathbb{R}^{+}, \dot{H}^{1}\left(\mathbb{R}^{3}\right)\right)} \\
& \leq C\left\|\sum_{j \leq \ell} v_{n}^{j}\right\|_{E_{\infty}^{\nu}}+C\left\|w_{n}^{\ell}\right\|_{E_{\infty}^{\nu}},
\end{aligned}
$$

by interpolation between $L^{\infty}\left(\mathbb{R}^{+}, \dot{H}^{1 / 2}\left(\mathbb{R}^{3}\right)\right)$ and $L^{2}\left(\mathbb{R}^{+}, \dot{H}^{3 / 2}\left(\mathbb{R}^{3}\right)\right)$. Proposition 3.4 and Proposition 3.2 enable us to infer that $\left(\sum_{j<\ell} v_{n}^{j}+w_{n}^{\ell}\right)$ is bounded in $L^{4}\left(\mathbb{R}^{+}, L^{6}\left(\mathbb{R}^{3}\right)\right)$, uniformly in $\ell$, so (3.21) and (3.22) yield the limits (3.17) and (3.18), and Proposition 3.3 is proved.

TOME $129-2001-\mathrm{N}^{\mathrm{O}} 2$ 
Proof of Proposition 3.4. - Lemma 3.1 implies that for $j$ large enough, the norm of $\varphi^{j}$ in $\dot{H}^{1 / 2}\left(\mathbb{R}^{3}\right)$ is smaller than $c \nu$, with notation (1.3). Then (1.4) enables us to infer that for $j$ large enough,

$$
V^{j} \in E_{\infty} \quad \text { and } \quad\left\|V^{j}\right\|_{E_{\infty}^{\nu}}^{2} \leq 2\left\|\varphi^{j}\right\|_{\dot{H}^{1 / 2}\left(\mathbb{R}^{3}\right)}^{2} .
$$

But (1.14) implies that the series of general term $\left\|\varphi^{j}\right\|_{\dot{H}^{1 / 2}\left(\mathbb{R}^{3}\right)}^{2}$ is convergent, therefore Proposition 3.4 follows.

3.2.3. Proof of Theorem 2 (i)-(ii): the general case. - Let us consider a sequence $\left(\varphi_{n}\right)$ of divergence free vector fields, bounded in $\dot{H}^{1 / 2}\left(\mathbb{R}^{3}\right)$, and let us define, with the notation of Theorem 1 ,

$$
v_{n} \stackrel{\text { def }}{=} N S\left(\varphi_{n}\right) \text { and } V^{j} \stackrel{\text { def }}{=} N S\left(\varphi^{j}\right) \text {. }
$$

We start by defining a sequence $\left(t_{n}\right) \in\left(\mathbb{R}^{+} \cup\{+\infty\}\right)^{\mathbb{N}}$ such that

$$
\left\|N S\left(\varphi_{n}\right)\right\|_{E_{t_{n}}^{\nu}} \text { is bounded. }
$$

The existence of $\left(t_{n}\right)$ is due to the fact that the application

$$
t \mapsto\left\|N S\left(\varphi_{n}\right)(t, \cdot)\right\|_{\dot{H}^{1 / 2}\left(\mathbb{R}^{3}\right)}^{2}+2 \nu\left\|N S\left(\varphi_{n}\right)\right\|_{L^{2}\left([0, t], \dot{H}^{3 / 2}\left(\mathbb{R}^{3}\right)\right)}^{2}
$$

is continuous on the time interval $\left[0, T_{*}\left(v_{n}\right)\left[\right.\right.$, where $T_{*}\left(v_{n}\right) \in \mathbb{R}^{+} \cup\{+\infty\}$ is the life span of $v_{n}$, and uniformly bounded at $t=0$ by $\sup _{n \in \mathbb{N}}\left\|\varphi_{n}\right\|_{\dot{H}^{1 / 2}\left(\mathbb{R}^{3}\right)}$. So for any real number $A>\sup _{n \in \mathbb{N}}\left\|\varphi_{n}\right\|_{\dot{H}^{1 / 2}\left(\mathbb{R}^{3}\right)}$ and any $n \in \mathbb{N}$, one can find a time $t_{n}(A)<T_{*}\left(v_{n}\right)$ such that

$$
\left\|N S\left(\varphi_{n}\right)\right\|_{E_{t_{n}(A)}^{\nu}} \leq A \text {. }
$$

Note that nothing prevents $t_{n}(A)$ from going to zero as $n$ goes to infinity.

Now let us prove that decomposition (1.18) holds, as well as properties (1.17) and (1.19). We start by writing the decomposition (1.11) of Theorem 1 , which reads

$$
\forall \ell \in \mathbb{N}, \quad \forall n \in \mathbb{N}, \quad \varphi_{n}(x)=\sum_{j=0}^{\ell} \varphi_{n}^{j}(x)+\psi_{n}^{\ell}(x),
$$

with notation (1.15) and (1.16), and with properties (1.12), (1.13), and (1.14).

Then the limit (1.12) enables us to define an integer $\ell_{0}$ in such a way that

$$
\limsup _{n \rightarrow \infty}\left\|\psi_{n}^{\ell_{0}}\right\|_{L^{3}\left(\mathbb{R}^{3}\right)} \leq \frac{1}{2} \delta \nu,
$$

where $\delta$ is the constant of Proposition A.1. Then in particular

$$
\left(N S\left(\psi_{n}^{\ell_{0}}\right)\right) \text { is bounded in } E_{\infty},
$$

BULLETIN DE LA SOCIÉTÉ MATHÉMATIQUE DE FRANCE 
by estimate (A.1) of Proposition A.1, since $\left\|\psi_{n}^{\ell_{0}}\right\|_{\dot{H}^{1 / 2}\left(\mathbb{R}^{3}\right)}$ is bounded according to (1.14).

Now recall that $L^{3}\left(\mathbb{R}^{3}\right)$ is an admissible space in the sense of Definition 1 , according to Example 2 given in the introduction; but Proposition A.1 implies that any function in $\dot{H}^{1 / 2}\left(\mathbb{R}^{3}\right)$ whose $L^{3}$ norm is smaller than $\delta \nu$ is in fact contained in $\mathcal{D}_{\infty}$, so it follows that

$$
\frac{1}{2} \delta \nu<C_{N S}^{L^{3}}
$$

with notation (1.9). So we can apply the result of Theorem 2 (iii) which yields

$$
N S\left(\psi_{n}^{\ell_{0}}\right)(t, x)=\sum_{j=\ell_{0}+1}^{\ell} \frac{1}{h_{n}^{j}} V^{j}\left(\frac{t}{\left(h_{n}^{j}\right)^{2}}, \frac{x-x_{n}^{j}}{h_{n}^{j}}\right)+w_{n}^{\ell}(t, x)+\tilde{r}_{n}^{\ell}(t, x),
$$

with the orthogonality property (1.13), the limit (3.2) for $w_{n}^{\ell}$, and where we have noted $V^{j} \stackrel{\text { def }}{=} N S\left(\varphi^{j}\right)$ for $j \geq \ell_{0}+1$. Furthermore, $V^{j}$ is an element of $E_{\infty}$ and

$$
\limsup _{\ell \rightarrow \infty} \operatorname{limsu}_{n \rightarrow \infty} \tilde{r}_{n}^{\ell}=0 \quad \text { in } E_{\infty}
$$

Note that in particular $(1.17)$ is proved, choosing $J \stackrel{\text { def }}{=}\left\{0, . ., \ell_{0}\right\}$.

So defining $V^{j} \stackrel{\text { def }}{=} N S\left(\varphi^{j}\right)$ for $j \leq \ell_{0}$, we can write for any $n \in \mathbb{N}$ and any $t \leq \min \left(\tau_{n}, t_{n}\right)$, where recall that $\tau_{n} \stackrel{\text { def }}{=} \min _{j \in J}\left(h_{n}^{j}\right)^{2} T^{j}$,

$$
\begin{aligned}
& N S\left(\varphi_{n}\right)(t, x) \\
& \quad=\sum_{j=0}^{\ell} \frac{1}{h_{n}^{j}} V^{j}\left(\frac{t}{\left(h_{n}^{j}\right)^{2}}, \frac{x-x_{n}^{j}}{h_{n}^{j}}\right)+w_{n}^{\ell}(t, x)+\tilde{r}_{n}^{\ell}(t, x)+\bar{r}_{n}^{\ell_{0}}(t, x) .
\end{aligned}
$$

The restriction $t \leq \min \left(\tau_{n}, t_{n}\right)$ is of course for the terms $N S\left(\varphi_{n}\right)$ and

$$
v_{n}^{j} \stackrel{\text { def }}{=} \frac{1}{h_{n}^{j}} V^{j}\left(\frac{t}{\left(h_{n}^{j}\right)^{2}}, \frac{x-x_{n}^{j}}{h_{n}^{j}}\right)
$$

in (3.30) to be defined. Then all we have to prove is that in (3.24), one can take

$$
t_{n}=\tau_{n},
$$

as well as the fact that (1.13) holds with the additional couples $\left(h_{n}^{j}, x_{n}^{j}\right)$ for $j \leq \ell_{0}$, and that

$$
\limsup _{n \rightarrow \infty}\left\|\bar{r}_{n}^{\ell_{0}}\right\|_{E_{\tau_{n}}^{\nu}}=0 \text {. }
$$

Then the theorem will be proved with $r_{n}^{\ell} \stackrel{\text { def }}{=} \tilde{r}_{n}^{\ell}+\bar{r}_{n}^{\ell_{0}}$.

The orthogonality of the couples $\left(h_{n}^{j}, x_{n}^{j}\right)$ for all integers $j$ is an obvious consequence of the orthogonality of the decomposition of the initial data due to Theorem 1, so let us check the two other points, (3.31) and (3.32). In fact

TOME $129-2001-\mathrm{N}^{\mathrm{O}} 2$ 
the result (3.31) is a consequence of (3.32) due to (3.30): if (3.32) is proved, then in particular all the terms in the right-hand side have a bounded $E_{\tau_{n}}^{\nu}$ norm, which implies that the same goes for $\left(v_{n}\right)$.

For convenience, let us re-order the functions $v_{n}^{j}$, for $j \leq \ell_{0}$, in such a way that, for $n$ large enough,

$$
\forall j \leq k \leq \ell_{0}, \quad\left(h_{n}^{j}\right)^{2} T_{*}^{j} \leq\left(h_{n}^{k}\right)^{2} T_{*}^{k} .
$$

We decide that if $T_{*}^{j}=+\infty$, then $\left(h_{n}^{j}\right)^{2} T_{*}^{j}=+\infty$ for every integer $n$. Recall that $T_{*}^{j}=+\infty$ does not imply $\varphi^{j} \in \mathcal{D}_{\infty}$, so if $T_{*}^{j}=+\infty$ for all $j$, and if there exists $j_{0}$ such that $\varphi^{j_{0}} \notin \mathcal{D}_{\infty}$, then we choose the first index 0 in such a way that $\varphi^{0}$ is not in $\mathcal{D}_{\infty}$. Note that $\left(h_{n}^{j}\right)^{2} T_{*}^{j}$ is the life span of $v_{n}^{j}$.

Now let us define the following scaling operator: for any vector field $f$, we define

$$
\forall j \in \mathbb{N}, \quad S_{j} f(s, y) \stackrel{\text { def }}{=} h_{n}^{j} f\left(\left(h_{n}^{j}\right)^{2} s, x_{n}^{j}+h_{n}^{j} y\right) .
$$

Note that

$$
\forall(j, n) \in \mathbb{N}^{2}, \quad S_{j} v_{n}^{j}=V^{j} .
$$

We then define, for every integer $\ell$,

$$
\begin{gathered}
\forall j \leq \ell, \quad V_{n}^{j, 0} \stackrel{\text { def }}{=} S_{0} v_{n}^{j}, \quad \bar{R}_{n}^{\ell_{0}, 0} \stackrel{\text { def }}{=} S_{0} \bar{r}_{n}^{\ell_{0}}, \\
W_{n}^{\ell, 0} \stackrel{\text { def }}{=} S_{0} w_{n}^{\ell}, \quad \widetilde{R}_{n}^{\ell, 0} \stackrel{\text { def }}{=} S_{0} \tilde{r}_{n}^{\ell}, \quad \text { and } \quad V_{n}^{0} \stackrel{\text { def }}{=} S_{0} v_{n} .
\end{gathered}
$$

Then the function $\bar{R}_{n}^{\ell_{0}, 0}$ satisfies the following system, similarly to (3.11):

$$
\left\{\begin{array}{l}
\partial_{s} \bar{R}_{n}^{\ell_{0}, 0}+P\left(\bar{R}_{n}^{\ell_{0}, 0} \cdot \nabla \bar{R}_{n}^{\ell_{0}, 0}\right)-\nu \Delta \bar{R}_{n}^{\ell_{0}, 0}+Q\left(\bar{R}_{n}^{\ell_{0}, 0}, F_{n}^{\ell, 0}\right)=G_{n}^{\ell, 0} \\
\bar{R}_{n \mid s=0}^{\ell_{0}, 0}=0
\end{array}\right.
$$

where

$$
F_{n}^{\ell, 0} \stackrel{\text { def }}{=} \sum_{j \leq \ell} V_{n}^{j, 0}+W_{n}^{\ell, 0}+\widetilde{R}_{n}^{\ell, 0}
$$

and

$$
\begin{aligned}
G_{n}^{\ell, 0} \stackrel{\text { def }}{=}-\frac{1}{2} \sum_{\substack{j \neq k \\
(j, k) \in\{0, . ., \ell\}^{2}}} Q\left(V_{n}^{j, 0}, V_{n}^{k, 0}\right) & -\sum_{j \leq \ell} Q\left(V_{n}^{j, 0}, W_{n}^{\ell, 0}+\widetilde{R}_{n}^{\ell, 0}\right) \\
& -\frac{1}{2} Q\left(W_{n}^{\ell, 0}, 2 \widetilde{R}_{n}^{\ell, 0}+W_{n}^{\ell, 0}\right) .
\end{aligned}
$$

Now let $T_{0}$ be any real number smaller than $T_{*}^{0}$. Then $V_{n}^{j, 0}=V^{0}$ is bounded in $E_{T^{0}}$, and according to (3.33) we have

$$
\forall j \in \mathbb{N}, \quad\left\|v_{n}^{j}\right\|_{E_{\left(h_{n}^{0}\right)^{2} T^{0}}} \text { is bounded, }
$$


since $\left(h_{n}^{0}\right)^{2} T^{0}<\left(h_{n}^{j}\right)^{2} T_{*}^{j}$ for $j \in\left\{0, . ., \ell_{0}\right\}$, and $v_{n}^{j}$ is bounded in $E_{\infty}$ for $j \geq \ell_{0}+1$. Moreover (3.38) can also be written

$$
\forall j \in \mathbb{N}, \quad\left(V_{n}^{j, 0}\right) \quad \text { is bounded in } E_{T^{0}} .
$$

We have the following proposition.

Proposition 3.5. - With notation (3.36) and (3.37), we have the following results: the sequence $\left(F_{n}^{\ell, 0}\right)$ is bounded in the space $E_{T^{0}}$ uniformly in $\ell$, and

$$
\lim _{\ell \rightarrow \infty} \limsup _{n \rightarrow \infty}\left\|G_{n}^{\ell, 0}\right\|_{L^{2}\left(\left[0, T^{0}\right], \dot{H}^{-1 / 2}\left(\mathbb{R}^{3}\right)\right)}=0 .
$$

Let us postpone the proof of Proposition 3.5 and finish the proof of the theorem. As in the previous section, Propositions 3.5 and A.2 imply that

$$
\limsup _{n \rightarrow \infty}\left\|\bar{R}_{n}^{\ell_{0}, 0}\right\|_{E_{T^{0}}^{\nu}}=0,
$$

and that proves (3.32) after a rescaling. Then as noticed above, the result (3.31) is simply due to the fact that all the terms in the decomposition of $v_{n}$ written in (3.30) are bounded in $E_{\left(h_{n}^{0}\right)^{2} T^{0}}$. That proves Theorem 2 (i).

Finally let us prove Theorem 2 (ii): suppose that there exists a time $T$ in $\mathbb{R}^{+} \cup\{+\infty\}$ such that $\left(v_{n}\right)$ is bounded in $L^{2}\left([0, T], \dot{H}^{3 / 2}\left(\mathbb{R}^{3}\right)\right)$. If for every integer $j$, the function $\varphi^{j}$ is in $\mathcal{D}_{\infty}$, then the result is proved. If not, then in particular we have $\varphi^{0} \notin \mathcal{D}_{\infty}$, with the re-ordering (3.33). In the computations above, we were free of our choice of the time $T^{0}$ provided $T^{0}<T_{*}^{0}$, so let us choose $T^{0}$ in the following way. We have, according to (3.30),

$$
\sum_{j=0}^{\ell_{0}} v_{n}^{j}+\bar{r}_{n}^{\ell_{0}}=v_{n}-N S\left(\psi_{n}^{\ell_{0}}\right)
$$

and since the sequence $\left(N S\left(\psi_{n}^{\ell_{0}}\right)\right)$ is bounded in $E_{\infty}$ by $(3.27)$ and $\left(v_{n}\right)$ is bounded in the space $L^{2}\left([0, T], \dot{H}^{3 / 2}\left(\mathbb{R}^{3}\right)\right)$, it follows that

$$
\sum_{j=0}^{\ell_{0}} v_{n}^{j}+\bar{r}_{n}^{\ell_{0}} \quad \text { is bounded in } \quad L^{2}\left([0, T], \dot{H}^{3 / 2}\left(\mathbb{R}^{3}\right)\right) .
$$

Then (1.2), and the remark after (1.8) if $T_{*}^{0}=+\infty$, enable us to choose $T^{0}<T_{*}^{0}$ such that

$$
\left\|V^{0}\right\|_{L^{2}\left(\left[0, T^{0}\right], \dot{H}^{3 / 2}\left(\mathbb{R}^{3}\right)\right)} \geq 2 \sup _{n \in \mathbb{N}}\left\|\sum_{j=0}^{\ell_{0}} v_{n}^{j}+\bar{r}_{n}^{\ell_{0}}\right\|_{L^{2}\left([0, T], \dot{H}^{3 / 2}\left(\mathbb{R}^{3}\right)\right)},
$$

which in turn yields

$$
\sum_{j=0}^{\ell_{0}}\left\|V_{n}^{j, 0}\right\|_{L^{2}\left(\left[0, T^{0}\right], \dot{H}^{3 / 2}\left(\mathbb{R}^{3}\right)\right)}^{2} \geq 4 \sup _{n \in \mathbb{N}}\left\|\sum_{j=0}^{\ell_{0}} v_{n}^{j}+\bar{r}_{n}^{\ell_{0}}\right\|_{L^{2}\left([0, T], \dot{H}^{3 / 2}\left(\mathbb{R}^{3}\right)\right)}^{2} .
$$

TOME $129-2001-\mathrm{N}^{\mathrm{O}} 2$ 
But Proposition 2.1 implies, after a rescaling, that

$$
\left\|\sum_{j=0}^{\ell_{0}} V_{n}^{j, 0}\right\|_{L^{2}\left(\left[0, T^{0}\right], \dot{H}^{3 / 2}\left(\mathbb{R}^{3}\right)\right)}^{2}=\sum_{j=0}^{\ell_{0}}\left\|V_{n}^{j, 0}\right\|_{L^{2}\left(\left[0, T^{0}\right], \dot{H}^{3 / 2}\left(\mathbb{R}^{3}\right)\right)}^{2}+o(1),
$$

when $n$ goes to infinity. So (3.40) implies that for any $\varepsilon>0$ and for $n$ large enough we have

$$
\begin{aligned}
& \sum_{j=0}^{\ell_{0}}\left\|V_{n}^{j, 0}\right\|_{L^{2}\left(\left[0, T^{0}\right], \dot{H}^{3 / 2}\left(\mathbb{R}^{3}\right)\right)}^{2} \\
& \leq \varepsilon+2 \sup _{n \in \mathbb{N}}\left\|\bar{R}_{n}^{\ell_{0}, 0}+\sum_{j=0}^{\ell_{0}} V_{n}^{j, 0}\right\|_{L^{2}\left(\left[0, T^{0}\right], \dot{H}^{3 / 2}\left(\mathbb{R}^{3}\right)\right)}^{2} \\
& \leq \varepsilon+2 \sup _{n \in \mathbb{N}}\left\|\bar{r}_{n}^{\ell_{0}}+\sum_{j=0}^{\ell_{0}} v_{n}^{j}\right\|_{L^{2}\left(\left[0,\left(h_{n}^{0}\right)^{2} T^{0}\right], \dot{H}^{3 / 2}\left(\mathbb{R}^{3}\right)\right)}^{2}
\end{aligned}
$$

The only way not to contradict (3.41) is to have $T \leq\left(h_{n}^{0}\right)^{2} T^{0}$, which proves Theorem 2 (ii).

Proof of Proposition 3.5. - The proof is very similar to the proof of Proposition 3.3 in the previous section, so we shall not go into too much detail. Let us start by noticing that the term $\widetilde{R}_{n}^{\ell, 0}$ satisfies the same estimates as $W_{n}^{\ell, 0}$, and in particular the limit (3.2), by Sobolev embeddings; so we shall forget it in the following.

Let us start by considering the term $F_{n}^{\ell, 0}$ : Proposition 3.4, along with the orthogonality properties (2.1) and (2.2), implies in particular that the sequence $\left\|\sum_{\ell_{0}+1}^{\ell} v_{n}^{j}\right\|_{E_{\infty}^{\nu}}^{2}$ is bounded independently of $\ell$; moreover we know from (3.38) that $\left(v_{n}^{j}\right)$ is bounded in $E_{\left(h_{n}^{0}\right)^{2} T^{0}}$ for $j \leq \ell_{0}$, so we get finally

$$
\forall \ell \in \mathbb{N}, \quad\left\|\sum_{j \leq \ell} v_{n}^{j}\right\|_{E_{\left(h_{n}^{0}\right)^{2} T^{0}}^{\nu}} \text { is bounded uniformly in } \ell,
$$

and the result is proved for $F_{n}^{\ell, 0}$ since $\left\|w_{n}^{\ell}\right\|_{E_{\infty}^{\nu}}$ is bounded uniformly in $\ell$ according to Proposition 3.2.

For the limit of $G_{n}^{\ell, 0}$, it is enough to prove the following results, after rescaling: for all $(j, k) \in\{0, . ., \ell\}^{2}$, with $j \neq k$,

(3.43) $\lim _{n \rightarrow \infty} Q\left(v_{n}^{j}, v_{n}^{k}\right)=0 \quad$ in $L^{4}\left(\left[0,\left(h_{n}^{0}\right)^{2} T^{0}\right], \dot{H}^{-1}\left(\mathbb{R}^{3}\right)\right)$

$$
\lim _{\ell \rightarrow \infty} \limsup _{n \rightarrow \infty} Q\left(\sum_{j \leq \ell} v_{n}^{j}, w_{n}^{\ell}\right)=0 \quad \text { in } \quad L^{4}\left(\left[0,\left(h_{n}^{0}\right)^{2} T^{0}\right], \dot{H}^{-1}\left(\mathbb{R}^{3}\right)\right) .
$$

Indeed, the limit of $Q\left(w_{n}^{\ell}, w_{n}^{\ell}\right)$ was proved in Proposition 3.3, and similar arguments to the case of $g_{n}^{\ell}$ above imply that $G_{n}^{\ell, 0}$ is bounded in $L^{4 / 3}\left(\left[0, T^{0}\right], L^{2}\left(\mathbb{R}^{3}\right)\right)$. Then the result follows by interpolation. 
The limit (3.43) follows from Proposition 2.1 exactly as (3.16), using (3.38). To prove (3.44), we follow the proof of (3.17): we have

$$
\begin{aligned}
& \left\|Q\left(\sum_{j \leq \ell} v_{n}^{j}, w_{n}^{\ell}\right)\right\|_{L^{4}\left(\left[0,\left(h_{n}^{0}\right)^{2} T^{0}\right], \dot{H}^{-1}\left(\mathbb{R}^{3}\right)\right)} \\
& \leq C\left\|\sum_{j \leq \ell} v_{n}^{j}\right\|_{L^{4}\left(\left[0,\left(h_{n}^{0}\right)^{2} T^{0}\right], L^{6}\left(\mathbb{R}^{3}\right)\right)}\left\|w_{n}^{\ell}\right\|_{L^{\infty}\left(\mathbb{R}^{+}, L^{3}\left(\mathbb{R}^{3}\right)\right)},
\end{aligned}
$$

and

$$
\forall n \in \mathbb{N}, \quad\left\|\sum_{j \leq \ell} v_{n}^{j}\right\|_{L^{4}\left(\left[0,\left(h_{n}^{0}\right)^{2} T^{0}\right], L^{6}\left(\mathbb{R}^{3}\right)\right)} \leq C\left\|\sum_{j \leq \ell} v_{n}^{j}\right\|_{E_{\left(h_{n}^{0}\right)^{2} T^{0}}^{\nu}} .
$$

Then (3.42) yields the result, and the proposition is proved.

3.3. Proof of Theorem 3. - We will keep the notation introduced in the course of the proof of Theorem 2, and we shall suppose in this section that additionnally to the assumptions of Theorem 2 , the sequence $\left(\varphi_{n}\right)$ is bounded in $L^{2}\left(\mathbb{R}^{3}\right)$.

The proof of the result (1.21) in Theorem 3 requires a more precise understanding of decomposition (1.11) given in Theorem 1. Let us therefore briefly recall how the scales $h_{n}^{j}$ are determined in [16]: a preliminary result in [16] states that any bounded sequence in $\dot{H}^{1 / 2}\left(\mathbb{R}^{3}\right)$ can be written, up to a subsequence, as

$$
\forall x \in \mathbb{R}^{3}, \quad \forall \ell \in \mathbb{N}, \quad \varphi_{n}(x)=\sum_{j=0}^{\ell} \widetilde{\varphi}_{n}^{j}(x)+\widetilde{\psi}_{n}^{\ell}(x),
$$

where $\lim _{\ell \rightarrow \infty} \lim \sup _{n \rightarrow \infty}\left\|\widetilde{\psi}_{n}^{\ell}\right\|_{L^{3}\left(\mathbb{R}^{3}\right)}=0$, and where each $\widetilde{\varphi}_{n}^{j}$ is bounded in $\dot{H}^{1 / 2}\left(\mathbb{R}^{3}\right)$ and strictly $h_{n}^{j}$-oscillatory, in the following sense.

Definition 2. - Let $\left(h_{n}\right)$ be a sequence in $\left(\mathbb{R}^{+} \backslash\{0\}\right)^{\mathbb{N}}$, and let $\left(\phi_{n}\right)$ be a bounded sequence in $\dot{H}^{1 / 2}\left(\mathbb{R}^{3}\right)$. Then $\left(\phi_{n}\right)$ is $h_{n}$-oscillatory if

$$
\lim _{R \rightarrow \infty} \limsup _{n \rightarrow \infty} \int_{h_{n}|\xi| \geq R}|\xi| \times\left|\widehat{\varphi_{n}}(\xi)\right|^{2} \mathrm{~d} \xi=0
$$

The sequence $\left(\phi_{n}\right)$ is strictly $h_{n}$-oscillatory if it also satisfies

$$
\lim _{\varepsilon \rightarrow 0} \limsup _{n \rightarrow \infty} \int_{h_{n}|\xi| \leq \varepsilon}|\xi| \times\left|\widehat{\varphi_{n}}(\xi)\right|^{2} \mathrm{~d} \xi=0 .
$$

Note that the sequences $\left(\widetilde{\varphi}_{n}^{j}\right)$ are of course also supposed not to converge strongly to zero in $\dot{H}^{1 / 2}\left(\mathbb{R}^{3}\right)$ as $n$ goes to infinity, and the sequences $\left(h_{n}^{j}\right)$ are orthogonal in the sense of (1.13); the cores of concentration $x_{n}^{j}$ in decomposition (1.11) are then extracted from each $\widetilde{\varphi}_{n}^{j}$ successively, and the profiles 
are determined at the same time. More precisely, one can write (see [16]) for every $(j, n) \in \mathbb{N}^{2}$ and every $x \in \mathbb{R}^{3}$, up to the extraction of a subsequence,

$$
\widetilde{\varphi}_{n}^{j}(x)=\sum_{k=0}^{\ell} \frac{1}{h_{n}^{j}} \varphi_{n}^{j, k}\left(\frac{x-x_{n}^{j, k}}{h_{n}^{j}}\right)+\psi_{n}^{j, \ell},
$$

with $\lim _{\ell \rightarrow \infty} \lim \sup _{n \rightarrow \infty}\left\|\psi_{n}^{j, \ell}\right\|_{L^{3}\left(\mathbb{R}^{3}\right)}=0$.

Finally it is proved in [16] that if $\left(\varphi_{n}\right)$ is bounded in $\dot{H}^{1 / 2}\left(\mathbb{R}^{3}\right) \cap L^{2}\left(\mathbb{R}^{3}\right)$, then for every integer $j,\left(\widetilde{\varphi}_{n}^{j}\right)$ is also bounded in $\dot{H}^{1 / 2}\left(\mathbb{R}^{3}\right) \cap L^{2}\left(\mathbb{R}^{3}\right)$ (see for instance Remark 2.10, formula (2.20), of [16]). So the result (1.21) on the limit of the sequence $\left(h_{n}^{j}\right)$ will be obtained if we prove the following lemma.

Lemma 3.6. - Let $\left(h_{n}\right)$ be a sequence in $\left(\mathbb{R}^{+} \backslash\{0\}\right)^{\mathbb{N}}$, converging towards infinity with $n$, and let $\left(\Phi_{n}\right)$ be a $h_{n}$-oscillatory sequence of functions, in the sense of Definition 2, bounded in $\dot{H}^{1 / 2}\left(\mathbb{R}^{3}\right)$. Suppose that $\left(\Phi_{n}\right)$ is bounded in $L^{2}\left(\mathbb{R}^{3}\right)$. Then $\Phi_{n}$ converges strongly to zero in $\dot{H}^{1 / 2}\left(\mathbb{R}^{3}\right)$.

Proof of Lemma 3.6. - Let us suppose that $\Phi_{n}$ does not converge strongly to zero in $\dot{H}^{1 / 2}\left(\mathbb{R}^{3}\right)$, and let us prove that $\left(\Phi_{n}\right)$ is not bounded in $L^{2}\left(\mathbb{R}^{3}\right)$. In that case, up to a subsequence, there exists $C_{0}>0$ such that $\left\|\Phi_{n}\right\|_{\dot{H}^{1 / 2}\left(\mathbb{R}^{3}\right)}>C_{0}$ for any $n$ large enough. Then (3.46) enables us to choose a real number $R_{0}$ such that

$$
\limsup _{n \rightarrow \infty} \int_{h_{n}|\xi| \geq R_{0}}|\xi| \times\left|\widehat{\Phi_{n}}(\xi)\right|^{2} \mathrm{~d} \xi \leq \frac{C_{0}}{2}
$$

But we have

$$
\begin{aligned}
\left\|\Phi_{n}\right\|_{L^{2}\left(\mathbb{R}^{3}\right)}^{2} & \geq \frac{h_{n}}{R_{0}} \int_{h_{n}|\xi| \leq R_{0}}|\xi| \times\left|\widehat{\Phi_{n}}(\xi)\right|^{2} \mathrm{~d} \xi \\
& \geq \frac{h_{n}}{R_{0}}\left(\left\|\Phi_{n}\right\|_{\dot{H}^{1 / 2}\left(\mathbb{R}^{3}\right)}^{2}-\int_{h_{n}|\xi| \geq R_{0}}|\xi| \times\left|\widehat{\Phi_{n}}(\xi)\right|^{2} \mathrm{~d} \xi\right),
\end{aligned}
$$

so (3.48) implies that

$$
\left\|\Phi_{n}\right\|_{L^{2}\left(\mathbb{R}^{3}\right)} \geq h_{n} \frac{C_{0}}{2 R_{0}}
$$

which yields the result, since $\left(h_{n}\right)$ is supposed to converge towards infinity with $n$.

That lemma implies that under the assumptions of Theorem 3, there are no large scales in the decomposition (3.45), and (1.21) is proved.

Finally let us prove (1.22): we suppose that $\left(v_{n}\right)$ is bounded in the space $L^{2}\left([0, T], \dot{H}^{3 / 2}\left(\mathbb{R}^{3}\right)\right)$, and we define

$$
J \stackrel{\text { def }}{=}\left\{j \leq \ell_{0} \mid h_{n}^{j}=1\right\},
$$

BULletin DE LA SOCIÉtÉ MATHÉMATIQUE DE FRANCE 
where $\ell_{0}$ was defined in (3.26). If $j$ is in $\mathbb{N} \backslash J$, then either $j \geq \ell_{0}+1$ and then $\varphi^{j} \in \mathcal{D}_{\infty}$, or $\lim _{n \rightarrow \infty} h_{n}^{j}=0$ in which case $\varphi^{j} \in \mathcal{D}_{\infty}$ according to Theorem 2 (ii). So $J$ satisfies (1.17). To prove (1.22), we recall that decomposition (3.30) implies that

$$
\left\{\begin{array}{l}
\text { for all } T^{0}<T_{*}^{0},\left\|v_{n}\right\|_{E_{\left(h_{n}^{0}\right)^{2} T^{0}}} \text { is bounded, } \\
\text { and if } \varphi^{0} \in \mathcal{D}_{\infty}, \text { then }\left\|v_{n}\right\|_{E_{\infty}^{\nu}} \text { is bounded }
\end{array}\right.
$$

with notation (3.33). Then two cases can occur: in the first case, we have $\lim _{n \rightarrow \infty} h_{n}^{0}=0$ and $\varphi^{0} \in \mathcal{D}_{\infty}$ according to Theorem 2 (ii). Then (3.49) implies that $\left(v_{n}\right)$ is bounded in $E_{\infty}$, and since $\varphi^{j} \in \mathcal{D}_{\infty}$, for all $j$, the result is proved. In the second case, we have $h_{n}^{0}=1$ so in particular $\left(v_{n}\right)$ is bounded in $L^{2}\left(\left[0, T^{0}\right], \dot{H}^{3 / 2}\left(\mathbb{R}^{3}\right)\right)$, which proves $(1.22)$ according to (1.2). So Theorem 3 is proved.

3.4. A remark in the case of periodic data. - If we suppose that the initial data is a sequence of periodic, mean free, divergence free vector fields $\left(\varphi_{n}\right)$, bounded in the space $\dot{H}^{1 / 2}\left(\mathbb{T}^{3}\right)$, where $\mathbb{T}^{3} \stackrel{\text { def }}{=}(\mathbb{R} / \mathbb{Z})^{3}$, then it is also bounded in $L^{2}\left(\mathbb{T}^{3}\right)$, since the $\dot{H}^{s}\left(\mathbb{T}^{3}\right)$ norm is defined by

$$
\|f\|_{\dot{H}^{s}\left(\mathbb{T}^{3}\right)}^{2} \stackrel{\text { def }}{=} \sum_{n \in \mathbb{Z}}|n|^{2 s} \times|\widehat{f}(n)|^{2},
$$

where $\widehat{f}(n)$ is the (discrete) Fourier transform of $f$. So the results of Theorem 3 apply. In particular, there are no large scales, and the scales equal to 1 are reduced to the weak limit only, since the cores $x_{n}^{j}$ are bounded when $h_{n}^{j}=1$. However, one must note that the profile decomposition of Theorem 1 must be slightly modified, for instance by multiplying the profiles $\varphi_{n}^{j}$ by truncations $\chi\left(x-x_{n}^{j}\right)$ where $\chi$ is supported in $[0,1]^{3}$. Similarly, one can decompose the solution $\left(v_{n}\right)$ also by multiplying the profiles in the decomposition of Theorem 2 by such truncations; the remainder induced by those truncations can be proved to be small in $E_{T}^{\nu}$, but that smallness is not uniform in $T$ even in the case of global solutions.

\section{Proof of the corollaries}

This section is devoted to the proof of the corollaries given in the introduction. We shall keep the notation of the previous sections.

4.1. Proof of Corollary 1. - As in [2] for the wave equation, Corollary 1 is proved by contradiction: let $\mathcal{A}$ be an admissible space, and suppose there exists a sequence of global solutions of (NS) in $E_{\infty}$, associated with a family

TOME $129-2001-\mathrm{N}^{\mathrm{O}} 2$ 
of initial data $\left(\varphi_{n}\right)$, bounded in a closed ball of $\mathcal{B}_{N S}^{\mathcal{A}}$ as defined in (1.10), such that

$$
\lim _{n \rightarrow \infty}\left\|N S\left(\varphi_{n}\right)\right\|_{E_{\infty}^{\nu}}=+\infty .
$$

We can apply Theorem 2 (iii) to $\left(\varphi_{n}\right)$. That implies in particular that $\left(N S\left(\varphi_{n}\right)\right)$ is bounded in $L^{\infty}\left(\mathbb{R}^{+}, \dot{H}^{1 / 2}\left(\mathbb{R}^{3}\right)\right) \cap L^{2}\left(\mathbb{R}^{+}, \dot{H}^{3 / 2}\left(\mathbb{R}^{3}\right)\right)$, since that holds for each term of the decomposition. The contradiction is obvious, and Corollary 1 is proved.

4.2. Proof of Corollary 2. - Let us prove that the mapping from data in $\mathcal{D}_{\infty}$ to the associate solution is Lipschitz on bounded subsets of $\mathcal{B}_{N S}^{\mathcal{A}}$, where $\mathcal{A}$ is an admissible space and $\mathcal{B}_{N S}^{\mathcal{A}}$ was defined in (1.10).

We start by proving the following .

Lemma 4.1. - Let $u_{0}$ and $v_{0}$ be two divergence free elements of $\mathcal{D}_{\infty}$, and define $u \stackrel{\text { def }}{=} N S\left(u_{0}\right)$. Then we have

$$
\frac{\mathrm{d}}{\mathrm{d} \varepsilon}\left(N S\left(u_{0}+\varepsilon v_{0}\right)\right)_{\mid \varepsilon=0}=v,
$$

where $v$ is the unique solution in $E_{\infty}$ of the following system:

$$
\left\{\begin{array}{l}
\partial_{t} v+P(v \cdot \nabla u+u \cdot \nabla v)-\nu \Delta v=0 \\
v_{\mid t=0}=v_{0}
\end{array}\right.
$$

where $P$ is the Leray projector onto divergence free vector fields. Moreover we have, for any $t \geq 0$,

$$
\begin{aligned}
\|v\|_{E_{t}^{\nu}} \leq C\left\|v_{0}\right\|_{\dot{H}^{1 / 2}\left(\mathbb{R}^{3}\right)}\{1 & \left.+\left(\int_{0}^{t}\|u(s)\|_{\dot{H}^{3 / 2}\left(\mathbb{R}^{3}\right)}^{2} \mathrm{~d} s\right)^{1 / 2}\right\} \\
& \times \exp \left(C \int_{0}^{t}\|u(s)\|_{\dot{H}^{3 / 2}\left(\mathbb{R}^{3}\right)}^{2} \mathrm{~d} s\right) .
\end{aligned}
$$

Proof of Lemma 4.1. - The fact that there is a unique solution to the linearized equation, which satisfies (4.2), is simply due to Proposition A.3 stated in the Appendix.

Now let $u^{\varepsilon}$ be defined by $u^{\varepsilon} \stackrel{\text { def }}{=} N S\left(u_{0}+\varepsilon v_{0}\right)$, and let us define

$$
\varepsilon r^{\varepsilon} \stackrel{\text { def }}{=} u^{\varepsilon}-u-\varepsilon v \text {. }
$$

We are going to prove that $\lim _{\varepsilon \rightarrow 0} r^{\varepsilon}=0$ in $E_{\infty}$, which clearly will imply the lemma. The function $r^{\varepsilon}$ satisfies the following equation:

with

$$
\left\{\begin{array}{l}
\partial_{t} r^{\varepsilon}+Q\left(r^{\varepsilon}, u\right)-\nu \Delta r^{\varepsilon}=f^{\varepsilon} \\
r_{\mid t=0}^{\varepsilon}=0
\end{array}\right.
$$

$$
f^{\varepsilon} \stackrel{\text { def }}{=}-\varepsilon P\left(v \cdot \nabla v+v \cdot \nabla r^{\varepsilon}+r^{\varepsilon} \cdot \nabla v+r^{\varepsilon} \cdot \nabla r^{\varepsilon}\right) .
$$

BULLETIN DE LA SOCiÉTÉ MATHÉMATIQUe DE FRANCE 
As a consequence, we can write, using Proposition A.3,

$$
\forall t \geq 0, \quad\left\|r^{\varepsilon}\right\|_{E_{t}^{\nu}} \leq C\left\|f^{\varepsilon}\right\|_{L_{t}^{2}\left(\dot{H}^{-1 / 2}\right)}\left(1+\|u\|_{L_{t}^{2}\left(\dot{H}^{3 / 2}\right)} \exp C\|u\|_{L_{t}^{2}\left(\dot{H}^{3 / 2}\right)}^{2}\right) .
$$

But the product rules in Sobolev spaces recalled in (3.19) imply that

$$
\left\|f^{\varepsilon}\right\|_{L_{t}^{2}\left(\dot{H}^{-1 / 2}\right)} \leq C \varepsilon\left(\|v\|_{E_{t}^{\nu}}^{2}+\|v\|_{E_{t}^{\nu}}\left\|r^{\varepsilon}\right\|_{E_{t}^{\nu}}+\left\|r^{\varepsilon}\right\|_{E_{t}^{\nu}}^{2}\right),
$$

so writing $X^{\varepsilon}(t) \stackrel{\text { def }}{=}\left\|r^{\varepsilon}\right\|_{E_{t}^{\nu}}$, we have

$$
\begin{aligned}
X^{\varepsilon}(t) \leq C \varepsilon & \left(\|v\|_{E_{t}^{\nu}}^{2}+\|v\|_{\left.E_{t}^{\nu} X^{\varepsilon}(t)+\left(X^{\varepsilon}(t)\right)^{2}\right)}\right. \\
& \times\left(1+\|u\|_{L_{t}^{2}\left(\dot{H}^{3 / 2}\right)} \exp C\|u\|_{L_{t}^{2}\left(\dot{H}^{3 / 2}\right)}^{2}\right) .
\end{aligned}
$$

Now let us recall that $u \in E_{\infty}$ by assumption; then if $C(u)$ denotes any constant depending on $\|u\|_{E_{\infty}^{\nu}}$, estimate (4.2) enables us to write

$$
\forall t \geq 0, \quad C(u) \varepsilon\left(X^{\varepsilon}(t)\right)^{2}-X^{\varepsilon}(t)(1-C(u) \varepsilon)+C(u) \varepsilon \geq 0 .
$$

But $X^{e}(t)$ is a continuous function and is equal to zero at time $t=0$; since $C(u) \varepsilon$ is the smallest root of the polynomial above, we conclude that

$$
\forall t \geq 0, \quad X^{\varepsilon}(t) \leq C(u) \varepsilon
$$

So the lemma is proved.

Finally Corollary 1 implies that in estimate $(4.2),\|u\|_{L^{2}\left([0, T], \dot{H}^{3 / 2}\left(\mathbb{R}^{3}\right)\right)}$ can be replaced by a function of $\left\|u_{0}\right\|_{\dot{H}^{1 / 2}\left(\mathbb{R}^{3}\right)}$, so Corollary 2 is proved.

\section{Appendix A}

\section{Some results on the Navier-Stokes equations}

In this final section, we shall present some results on the Navier-Stokes equations which have been used in the proofs. Those results are quite classical. We are also going to prove Proposition 1.1 stated in the introduction.

The first result is of the propagation of regularity type. It can be seen as a corollary of Theorem 3.4.2 of [3], but we present here a self-contained proof.

Proposition A.1. - There exists a constant $\delta$ such that the following is true. Let $v_{0} \in \dot{H}^{1 / 2}\left(\mathbb{R}^{3}\right)$ be a divergence free vector field, such that $\left\|v_{0}\right\|_{L^{3}\left(\mathbb{R}^{3}\right)}$ is smaller than $\delta \nu$. Then $v_{0} \in \mathcal{D}_{\infty}$, and if $v \stackrel{\text { def }}{=} N S\left(v_{0}\right)$, then for any $t \geq 0$ we have

$$
\|v(t)\|_{\dot{H}^{1 / 2}\left(\mathbb{R}^{3}\right)}^{2}+\nu \int_{0}^{t}\|\nabla v(s)\|_{\dot{H}^{1 / 2}\left(\mathbb{R}^{3}\right)}^{2} \mathrm{~d} s \leq\left\|v_{0}\right\|_{\dot{H}^{1 / 2}\left(\mathbb{R}^{3}\right)}^{2} .
$$

TOME $129-2001-\mathrm{N}^{\mathrm{O}} 2$ 
Proof of Proposition A.1. - It is known (see [10],[18]) that there exists a constant $c>0$ such that if $\left\|v_{0}\right\|_{L^{3}\left(\mathbb{R}^{3}\right)} \leq c \nu$, then $N S\left(v_{0}\right)$ is in $C^{0}\left(\mathbb{R}^{+}, L^{3}\left(\mathbb{R}^{3}\right)\right)$, and we have

$$
\forall t \geq 0, \quad\left\|N S\left(v_{0}\right)(t, \cdot)\right\|_{L^{3}\left(\mathbb{R}^{3}\right)} \leq C\left\|v_{0}\right\|_{L^{3}\left(\mathbb{R}^{3}\right)},
$$

where $C$ is a universal constant. Moreover, recall that $L^{3}\left(\mathbb{R}^{3}\right)$ is an admissible space in the sense of Definition 1, according to Example 2 of the introduction. So property (ii) of Definition 1 enables us to choose a constant $\delta \leq c$ such that if $\left\|v_{0}\right\|_{L^{3}\left(\mathbb{R}^{3}\right)} \leq \delta \nu$, then $v_{0} \in \mathcal{D}_{\infty}$. Now let us prove the estimate (A.1): an energy estimate in $\dot{H}^{1 / 2}\left(\mathbb{R}^{3}\right)$ for the function $v(t, \cdot)$ reads

$$
\frac{1}{2} \frac{\mathrm{d}}{\mathrm{d} t}\|v(t)\|_{\dot{H}^{1 / 2}\left(\mathbb{R}^{3}\right)}^{2}+\nu\|\nabla v(t)\|_{\dot{H}^{1 / 2}\left(\mathbb{R}^{3}\right)}^{2}=\left|(v \cdot \nabla v(t) \mid v(t))_{\dot{H}^{1 / 2}\left(\mathbb{R}^{3}\right)}\right| .
$$

But writing $\Lambda \stackrel{\text { def }}{=} \sqrt{-\Delta}$, we have

$$
(v \cdot \nabla v(t) \mid v(t))_{\dot{H}^{1 / 2}\left(\mathbb{R}^{3}\right)}=(v \cdot \nabla v(t) \mid \Lambda v(t))_{L^{2}\left(\mathbb{R}^{3}\right)},
$$

and Hölder's inequality yields

$$
\left|(v \cdot \nabla v(t) \mid \Lambda v(t))_{L^{2}\left(\mathbb{R}^{3}\right)}\right| \leq\|v(t)\|_{L^{3}\left(\mathbb{R}^{3}\right)} \times\|\nabla v(t)\|_{L^{3}\left(\mathbb{R}^{3}\right)}^{2} .
$$

The continuous embedding of $\dot{H}^{1 / 2}\left(\mathbb{R}^{3}\right)$ into $L^{3}\left(\mathbb{R}^{3}\right)$ enables us to infer that

$$
\frac{1}{2} \frac{\mathrm{d}}{\mathrm{d} t}\|v(t)\|_{\dot{H}^{1 / 2}\left(\mathbb{R}^{3}\right)}^{2}+\nu\|\nabla v(t)\|_{\dot{H}^{1 / 2}\left(\mathbb{R}^{3}\right)}^{2} \leq C\|v(t)\|_{L^{3}\left(\mathbb{R}^{3}\right)} \times\|\nabla v(t)\|_{\dot{H}^{1 / 2}\left(\mathbb{R}^{3}\right)}^{2},
$$
so finally choosing $\delta$ small enough, we have according to (A.2),

$$
\frac{\mathrm{d}}{\mathrm{d} t}\|v(t)\|_{\dot{H}^{1 / 2}\left(\mathbb{R}^{3}\right)}^{2}+\nu\|\nabla v(t)\|_{\dot{H}^{1 / 2}\left(\mathbb{R}^{3}\right)}^{2} \leq 0 .
$$

The proposition is proved.

Proposition A.2. - Let $T \in \mathbb{R}^{+} \cup\{+\infty\}$ and $\nu>0$ be fixed. There exists a constant $C$, depending on $\nu$ but not on $T$, such that the following is true. Let $\left(f_{n}\right)$ and $\left(g_{n}\right)$ be two families of vector fields, bounded in $E_{T}$ and in $L^{2}\left([0, T], \dot{H}^{-1 / 2}\left(\mathbb{R}^{3}\right)\right)$ respectively. If

$$
\sup _{n \in \mathbb{N}}\left\|g_{n}\right\|_{L^{2}\left([0, T], \dot{H}^{-1 / 2}\left(\mathbb{R}^{3}\right)\right)} \leq C \exp \left(-2 C \sup _{n \in \mathbb{N}}\left\|f_{n}\right\|_{E_{T}^{\nu}}^{4}\right),
$$

then there exists a unique solution in $E_{T}$ to the following system:

$$
\left\{\begin{array}{l}
\partial_{t} r_{n}+P\left(r_{n} \cdot \nabla r_{n}\right)-\nu \Delta r_{n}+Q\left(r_{n}, f_{n}\right)=g_{n}, \\
r_{n \mid t=0}=0
\end{array}\right.
$$

where $P$ is the Leray projector onto divergence free vector fields, and where

$$
Q(a, b) \stackrel{\text { def }}{=} P(a \cdot \nabla b+b \cdot \nabla a) .
$$

Moreover, we have, with notation (1.8), and writing

$$
L_{T}^{2}\left(\dot{H}^{-1 / 2}\left(\mathbb{R}^{3}\right)\right) \stackrel{\text { def }}{=} L^{2}\left([0, T], \dot{H}^{-1 / 2}\left(\mathbb{R}^{3}\right),\right.
$$

BULletin DE LA SOCiÉtÉ MATHÉMATIQUE DE FRANCE 


$$
\left\|r_{n}\right\|_{E_{T}^{\nu}} \leq C\left\|g_{n}\right\|_{L_{T}^{2}\left(\dot{H}^{-1 / 2}\left(\mathbb{R}^{3}\right)\right)}\left(1+\exp \left(C\left\|f_{n}\right\|_{E_{T}^{\nu}}^{4}\right)\right) .
$$

Proof of Proposition A.2. - Let us start by noticing that any function in $E_{T}$ is in $L^{4}\left([0, T], \dot{H}^{1}\left(\mathbb{R}^{3}\right)\right)$, by interpolation between $L^{\infty}\left([0, T], \dot{H}^{1 / 2}\left(\mathbb{R}^{3}\right)\right)$ and $L^{2}\left([0, T], \dot{H}^{3 / 2}\left(\mathbb{R}^{3}\right)\right)$. Then the proposition is a direct consequence of Lemma B.1 of [12]: formula (B.3) of [12] indeed states that

$$
\begin{aligned}
\left\|r_{n}\right\|_{L_{T}^{4}\left(\dot{H}^{1}\left(\mathbb{R}^{3}\right)\right)} \leq C\left(\left\|g_{n}\right\|_{L_{T}^{2}\left(\dot{H}^{-1 / 2}\left(\mathbb{R}^{3}\right)\right)}\right. & \left.+\left\|r_{n}\right\|_{L_{T}^{4}\left(\dot{H}^{1}\left(\mathbb{R}^{3}\right)\right)}^{2}\right) \\
& \times \exp \left(C\left\|f_{n}\right\|_{L_{T}^{4}\left(\dot{H}^{1}\left(\mathbb{R}^{3}\right)\right)}^{4}\right) .
\end{aligned}
$$

Then assumption (A.3) enables us to write, by superlinear bootstrap (see for instance [2], Lemma 2.2), that

$$
\left\|r_{n}\right\|_{L_{T}^{4}\left(\dot{H}^{1}\left(\mathbb{R}^{3}\right)\right)} \leq C\left\|g_{n}\right\|_{L_{T}^{2}\left(\dot{H}^{-1 / 2}\left(\mathbb{R}^{3}\right)\right)} \exp \left(C\left\|f_{n}\right\|_{L_{T}^{4}\left(\dot{H}^{1}\left(\mathbb{R}^{3}\right)\right)}^{4}\right) .
$$

But Lemma B.1 of [12] also implies that

so the result follows.

$$
\begin{aligned}
& \left\|r_{n}\right\|_{L_{T}^{\infty}\left(\dot{H}^{1 / 2}\left(\mathbb{R}^{3}\right)\right)}+\left\|r_{n}\right\|_{L_{T}^{2}\left(\dot{H}^{3 / 2}\left(\mathbb{R}^{3}\right)\right)} \leq C\left\|g_{n}\right\|_{L_{T}^{2}\left(\dot{H}^{-1 / 2}\left(\mathbb{R}^{3}\right)\right)} \\
& +C\left\|r_{n}\right\|_{L_{T}^{4}\left(\dot{H}^{1}\left(\mathbb{R}^{3}\right)\right)}\left(\left\|r_{n}\right\|_{L_{T}^{4}\left(\dot{H}^{1}\left(\mathbb{R}^{3}\right)\right)}+\left\|f_{n}\right\|_{L_{T}^{4}\left(\dot{H}^{1}\left(\mathbb{R}^{3}\right)\right)}\right)
\end{aligned}
$$

The following proposition is similar to the previous one, and is a direct consequence of Lemma B.1 of [12].

Proposition A.3. - Let $T \in \mathbb{R}^{+} \cup\{+\infty\}$ be fixed, and let $f_{n}$ and $g_{n}$ be two vector fields, bounded respectively in the space $L^{2}\left([0, T], \dot{H}^{3 / 2}\left(\mathbb{R}^{3}\right)\right)$, and in the space $L^{2}\left([0, T], \dot{H}^{-1 / 2}\left(\mathbb{R}^{3}\right)\right)$. Finally let $r_{n, 0}$ be a family of divergence free vector fields, bounded in $\dot{H}^{1 / 2}\left(\mathbb{R}^{3}\right)$. Then there exists a unique solution in $E_{T}$ to the following system:

$$
\left\{\begin{array}{l}
\partial_{t} r_{n}-\nu \Delta r_{n}+Q\left(r_{n}, f_{n}\right)=g_{n}, \\
r_{n \mid t=0}=r_{n, 0},
\end{array}\right.
$$

where $Q(a, b) \stackrel{\text { def }}{=} P(a \cdot \nabla b+b \cdot \nabla a)$. Moreover, we have

$$
\begin{aligned}
\left\|r_{n}\right\|_{E_{T}^{\nu}} \leq C & \left(\left\|r_{n, 0}\right\|_{\dot{H}^{1 / 2}}+\left\|g_{n}\right\|_{L_{T}^{2}\left(\dot{H}^{-1 / 2}\right)}\right) \\
& \times\left(1+\left\|f_{n}\right\|_{L_{T}^{2}\left(\dot{H}^{3 / 2}\right)} \exp \left(C\left\|f_{n}\right\|_{L_{T}^{2}\left(\dot{H}^{3 / 2}\right)}^{2}\right)\right),
\end{aligned}
$$

where $C$ is a constant which depends on $\nu$ but not on $T$.

Finally let us prove Proposition 1.1 stated in the introduction. We recall the statement below.

Proposition A.4. - Let $v_{0} \in H^{1 / 2}\left(\mathbb{R}^{3}\right)$ be a divergence free vector field, and let $T_{*}$ be the life span of $N S\left(v_{0}\right)$. If $v_{0} \notin \mathcal{D}_{\infty}$, then

$$
T_{*} \leq \frac{1}{\nu\left(C_{N S}^{\dot{H}^{1 / 2}}\right)^{4}}\left\|v_{0}\right\|_{L^{2}\left(\mathbb{R}^{3}\right)} .
$$

TOME $129-2001-\mathrm{N}^{\mathrm{O}} 2$ 
Proof of Proposition A.4. - The proof is also quite classical (see for instance [11] in a different context); as it is quite short, we recall it here for the convenience of the reader. So let us consider a divergence free vector field $v_{0} \in H^{1 / 2}\left(\mathbb{R}^{3}\right)$, and let us suppose that $v_{0} \notin \mathcal{D}_{\infty}$. Let $T_{*}$ be the life span of $v \stackrel{\text { def }}{=} N S\left(v_{0}\right)$. Then for all $T<T_{*}$, writing $\lambda(X)$ for the Lebesgue measure of $X$, we have

$$
\begin{aligned}
\lambda\left\{t \in \left[0, T\left[; C_{N S}^{\dot{H}^{1 / 2}}\right.\right.\right. & \left.\leq\|v(t, \cdot)\|_{\dot{H}^{1 / 2}\left(\mathbb{R}^{3}\right)}\right\} \\
\leq & \frac{1}{\left(C_{N S}^{\dot{H}^{1 / 2}}\right)^{4}} \int_{0}^{T}\|v(t, \cdot)\|_{\dot{H}^{1 / 2}\left(\mathbb{R}^{3}\right)}^{4} \mathrm{~d} t
\end{aligned}
$$

by the Bienaymé-Tchebytchev inequality. That yields

$$
T_{*} \leq \frac{1}{\left(C_{N S}^{\dot{H}^{1 / 2}}\right)^{4}}\|v\|_{L^{\infty}\left(\mathbb{R}^{+}, L^{2}\left(\mathbb{R}^{3}\right)\right)}^{2} \times\|v\|_{L^{2}\left(\mathbb{R}^{+}, \dot{H}^{1}\left(\mathbb{R}^{3}\right)\right)}^{2} \leq \frac{1}{\nu\left(C_{N S}^{\dot{H}^{1 / 2}}\right)^{4}}\left\|v_{0}\right\|_{L^{2}\left(\mathbb{R}^{3}\right)}^{4} .
$$

So the result is proved.

\section{BIBLIOGRAPHY}

[1] BAhouri (H.), GÉRARD (P.) - Concentration effects in critical nonlinear wave equations, in Geometrical Optics and Related Topics (F. Colombini and N. Lerner eds.), Progress in Nonlinear Differential Equations and Applications, 32, Birkhäuser, Boston, 1997, pp. 17-30.

[2] BAhouri (H.), GÉRARD (P.) - High frequency approximation of solutions to critical nonlinear wave equations, Amer. J. Math., 121 (1999), pp. 131175.

[3] Cannone (M.) - Ondelettes, paraproduits et Navier-Stokes, Diderot éd., Arts et Sciences, 1995.

[4] Chemin (J.-Y.) - Remarques sur l'existence globale pour le système de Navier-Stokes incompressible, SIAM J. Math. Anal., 23 (1992), pp. 20-28.

[5] Chemin (J.-Y.) - Fluides parfaits incompressibles, Astérisque, 230, 1995.

[6] Chemin (J.-Y.) - Théorèmes d'unicité pour le système de Navier-Stokes tridimensionnel, J. Anal. Math., 77 (1999), p. 27-50.

[7] Constantin (P.), Foias (C.) - Navier-Stokes Equations, Chicago University Press, 1988.

[8] Fujita (H.), Kato (T.) - On the Navier-Stokes initial value problem I, Archive Rat. Mech. Anal., 16 (1964), pp. 269-315.

[9] Furioli (G.) - Applications de l'analyse harmonique réelle à l'étude des équations de Navier-Stokes et de Schrödinger non linéaire, Thesis of Évry University, 1999. 
[10] Furioli (G.), Lemarié (P.-G.), Terraneo (E.) - Unicité des solutions mild des équations de Navier-Stokes dans $L^{3}\left(\mathbb{R}^{3}\right)$ et d'autres espaces limites, to appear in Revista Mat. Iberoamer. and Preprint of Évry University, 1997.

[11] Gallagher (I.) - The Tridimensional Navier-Stokes equations with almost bidimensional data: stability, uniqueness and life span, Inter. Math. Research Notices, 18 (1997), pp. 919-935.

[12] Gallagher (I.) - Applications of Schochet's methods to parabolic equations, J. Math. Pures Appl., 77 (1998), pp. 989-1054.

[13] Gallagher (I.) - Décomposition en profils des solutions des équations de Navier-Stokes, Séminaire Équations aux Dérivées Partielles, École Polytechnique, May 2000.

[14] Gallagher (I.), GÉRARd (P.) - Profile decomposition for the wave equation outside a convex obstacle, J. Math. Pures Appl., 80 (2001), pp. 1-49.

[15] GÉRARD (P.) - Oscillations and concentration effects in semilinear dispersive wave equations, J. Funct. Anal., 141 (1996), pp. 60-98.

[16] GÉRARD (P.) - Description du défaut de compacité de l'injection de Sobolev, ESAIM Contrôle Optimal et Calcul des Variations, 3 (1998), pp. 213-233 (electronic version: http://www.emath.fr/cocv/).

[17] Gérard (P.), Markowitch (P.), Mauser (N.), Poupaud (F.) - Homogenization limits and Wigner transforms, Comm. Pure Applied Math., 50 (1997), pp. 323-379.

[18] Kato (T.) - Strong $L^{p}$ solutions of the Navier-Stokes equations in $\mathbb{R}^{m}$ with applications to weak solutions, Math. Zeit. 187 (1984), pp. 471-480.

[19] KerAani (S.) - On the defect of compactness for the Strichartz estimates of the Schrödinger equations, Preprint, Université de Paris-Sud, 1999, and to appear in J. Diff. Equ.

[20] Koch (H.), TAtaru (D.) - Well-posedness for the Navier-Stokes equations, Adv. Math. 157 (2001), pp. 22-35.

[21] LERAY (J.) - Essai sur le mouvement d'un liquide visqueux emplissant l'espace, Acta Mat., 63 (1933), pp. 193-248.

[22] Peetre (J.) - New Thoughts on Besov Spaces, Duke University Mathematical Series, 1976.

[23] Planchon (F.) - Global strong solutions in Sobolev or Lebesgue spaces to the incompressible Navier-Stokes equations in $\mathbb{R}^{3}$, Ann. Inst. Henri Poincaré, 13 (1996), pp. 319-336.

[24] Stein (E.) - Harmonic Analysis, Princeton University Press, 1992.

[25] Triebel (H.) - Theory of Function Spaces, Monographs in Mathematics 78, Birkhauser Verlag, 1983. 